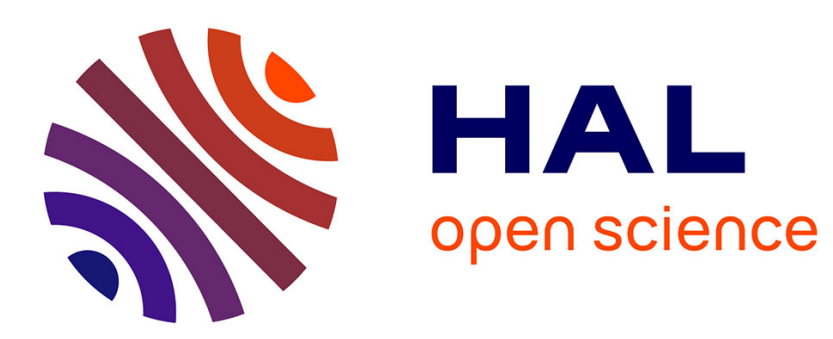

\title{
Towards nonlinear imperfect interface models including micro-cracks and smooth roughness
}

Serge Dumont, Frédéric Lebon, Maria Letizia Raffa, Raffaella Rizzoni

\section{To cite this version:}

Serge Dumont, Frédéric Lebon, Maria Letizia Raffa, Raffaella Rizzoni. Towards nonlinear imperfect interface models including micro-cracks and smooth roughness. Annals of Solid and Structural Mechanics, 2017, 9 (1-2), pp.13-27. 10.1007/s12356-017-0047-8 . hal-01697064

\section{HAL Id: hal-01697064 https://hal.science/hal-01697064}

Submitted on 29 Aug 2018

HAL is a multi-disciplinary open access archive for the deposit and dissemination of scientific research documents, whether they are published or not. The documents may come from teaching and research institutions in France or abroad, or from public or private research centers.
L'archive ouverte pluridisciplinaire HAL, est destinée au dépôt et à la diffusion de documents scientifiques de niveau recherche, publiés ou non, émanant des établissements d'enseignement et de recherche français ou étrangers, des laboratoires publics ou privés. 


\title{
Towards nonlinear imperfect interface models including micro-cracks and smooth roughness
}

\author{
S. Dumont ${ }^{1} \cdot$ F. Lebon ${ }^{2} \cdot$ M. L. Raffa ${ }^{3} \cdot$ R. Rizzoni ${ }^{4}$
}

\begin{abstract}
The present paper deals with a general asymptotic theory aimed at deriving some imperfect interface models starting from thin interphases. The novelty of this work consists in taking into account some non-standard constitutive behaviors for the interphase material. In particular, micro-cracks, surface roughness and geometrical nonlinearity are included into the general framework of the matched-asymptotic-expansion theory. The elastic equilibrium problem of a three-composite body comprising two elastic adherents and an adhesive interphase is investigated. Higher order interface models are derived within the cases of soft and hard interphase materials. Simple FEM-based numerical applications are also presented.
\end{abstract}

F. Lebon

lebon@1ma.cnrs-mrs.fr

S. Dumont

serge.dumont@unimes.fr

M. L. Raffa

maria-letizia.raffa@u-pec.fr

R. Rizzoni

rizzoni.raffaella@unife.it

1 Université de Nîmes, Institut Montpellierain Alexander Grothendieck, CNRS UMR 5149, CC 051, Place Eugène Bataillon 34090 Montpellier, France

2 Aix-Marseille Univ., CNRS, Centrale Marseille, LMA, Marseille, France

3 CNRS, Laboratoire Modélisation et Simulation Multi Echelle, MSME UMR 8208 CNRS, 61 avenue du Général de Gaulle, 94010 Créteil Cedex, France

4 Department of Engineering, University of Ferrara, via Saragat 1, 44122 Ferrara, Italy
Keywords Asymptotic analysis - Nonlinear constitutive equations $\cdot$ Roughness $\cdot$ Imperfect interface $\cdot$ Nonlinear contact laws

\section{Introduction}

Nowadays structural bonding is a very common technique in numerous industrial domains as aeronautics or civil engineering. This technology consists in an adhesive assembly of two bodies. The main function of this assembly is to transmit mechanical stresses in a given environment during the whole life of the structure. The technology's benefits are obvious: low costs, simplified industrial processes, increased lightness, etc. Conventional adhesives (epoxides, polyamides, acrylic, cyanoacrylates, etc.) come in the form of thin films. Therefore, it is very important to propose simple as well as precise analytical and numerical models to simulate and predict the behavior of thin adhesive layers. The present work aims at proposing a quite general methodology to describe the behavior of such a structure. Adhesive layers being often characterized by their low thickness, it seems natural to introduce an asymptotic model whose small parameter is the layers' smallest characteristic length $\varepsilon$. The problem of a thin adhesive joint is then classically replaced by a transmission problem with mechanical characteristics obtained from the mechanical and geometrical characteristics of the adhesive thin film (cf. [2, 12, 13, 15]).

In former papers by authors ([36, 38, 39, 45] and references therein), the illustrated methodology has been applied for a planar elastic thin adhesive interphase, possibly anisotropic. Furthermore, in [37] this asymptotic technique within the small strains context was coupled to micromechanical homogenization concepts and Hertzian 
theory in order to formulate a model of contact between smoothly-rough surfaces in non-sliding conditions.

In the present paper, several additional new features are introduced. Particularly, an overview of simple techniques accounting for (smooth) roughness, micro-damage (microcracks) and geometrical nonlinearities is presented. Two main novelties are presented: (1) roughness is introduced in the asymptotic formulation within the framework of finite strain $[36,39,44]$ as well as in the hard interface formulation; (2) two theories of micromechanical homogenization for micro-cracked materials, i.e., Kachanov's theory [30, $48]$ and dilute scheme [4, 35], are studied and compared.

The paper is organized as follows. Firstly, a review of soft and hard imperfect interface models in linear elasticity is presented in Sect. 2. Next, a smooth roughness at the contacting interfaces between the two adherents and the adhesive interphase in introduced in Sect. 3. A simple methodology of micromechanical homogenization able to take into account micro-damage is presented in Sect. 4. In Sect. 5, an interphase consisting of a nonlinear elastic material, the Saint Venant-Kirchhoff material model, is studied. Lastly, some simple numerical applications are proposed in Sect. 6 , to validate the proposed interface models.

\section{Soft and hard imperfect interface models in linear elasticity}

We begin by specifying what we understand by "soft" and "hard" interfaces. This notion is related to the stiffness ratio between the adhesive and the adherents. For example, in the case of two pieces of steel (with a stiffness close to $210 \mathrm{GPa}$ ) bonded by an araldite glue (a stiffness close to 2 $\mathrm{GPa}$ ), the interphase is considered as soft. On the contrary, if two samples of maple wood (the stiffness is close to 10
GPa) are bonded by an epoxy resin (the Young's modulus is close to $4 \mathrm{GPa}$ ), the interphase is considered as hard.

\subsection{The three-dimensional equilibrium problem}

A thin interphase, $B^{\varepsilon}$, joining two adherents, $\Omega_{ \pm}^{\varepsilon}$, is considered occupying a cylindrical region of small thickness and cross-section $S$, with $S$ an open bounded set in $\mathrm{IR}^{2}$ with a smooth boundary $\partial S$. Introduced the Cartesian frame $\left(O, x_{1}, x_{2}, x_{3}\right)$ with $\left(\mathbf{e}_{1}, \mathbf{e}_{2}, \mathbf{e}_{3}\right)$ the corresponding orthonormal basis, the domains $B^{\varepsilon}$ and $\Omega_{ \pm}^{\varepsilon}$ are defined as

$B^{\varepsilon}=\left\{\left(x_{1}, x_{2}, x_{3}\right) \in \Omega:-\frac{\varepsilon}{2}<x_{3}<\frac{\varepsilon}{2}\right\}$.

$\Omega_{ \pm}^{\varepsilon}=\left\{\left(x_{1}, x_{2}, x_{3}\right) \in \Omega: \pm x_{3}>\frac{\varepsilon}{2}\right\}$,

with $0<\varepsilon / h<<1$ and $h$ the characteristic length of $\Omega$. The surfaces between the adherents and the interphase of small thickness $\varepsilon$ are

$S_{ \pm}^{\varepsilon}=\left\{\left(x_{1}, x_{2}, x_{3}\right) \in \Omega: x_{3}= \pm \frac{\varepsilon}{2}\right\}$.

The geometrical configuration described above is shown in Fig. 1.

The adherents are subjected to a body force density $\mathbf{f}: \Omega^{\varepsilon}{ }_{ \pm} \mapsto R^{3}$ and to a surface force density $\mathbf{g}: \Gamma_{g}^{\varepsilon} \mapsto R^{3}$. Body forces are neglected in the adhesive.

On $\Gamma_{u}^{\varepsilon}=\partial \Omega^{\varepsilon} \backslash\left(\Gamma_{g}^{\varepsilon} \cup \partial \Omega^{\varepsilon} \cap \partial B^{\varepsilon}\right)$, homogeneous boundary conditions are prescribed:

$\mathbf{u}^{\varepsilon}=\mathbf{0} \quad$ on $\Gamma_{u}^{\varepsilon}$,

where $\mathbf{u}^{\varepsilon}: \Omega^{\varepsilon} \mapsto R^{3}$ is the displacement field defined on $\Omega^{\varepsilon}$. $\Gamma_{g}^{\varepsilon}$ is assumed to be located far from the interphase and the fields of the external forces are endowed with sufficient
Fig. 1 Geometrical configurations of the three-composite system within the a the reference model (interphase); $\mathbf{b}$ the rescaled model and $\mathbf{c}$ the limit model (interface)

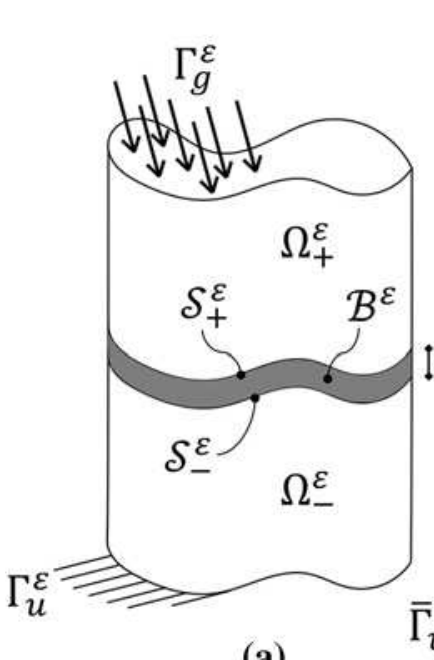

(a)

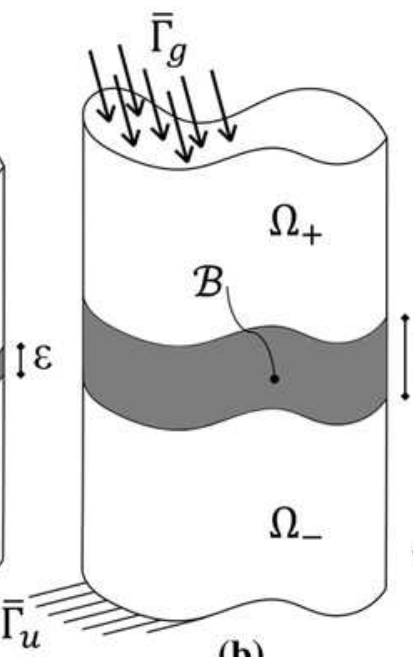

(b)

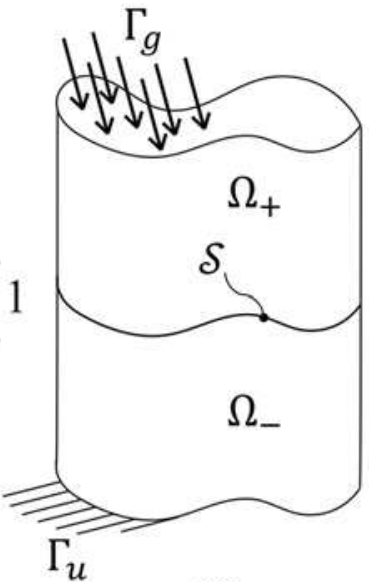

(c) 
regularity to ensure the existence of equilibrium configuration. In the following, the symbol (, ) means derivative. The equations governing the equilibrium problem of the composite structure are written as follows:

$\begin{cases}\sigma_{i j, j}^{\varepsilon}+f_{i}=0 & \text { in } \Omega_{ \pm}^{\varepsilon}, \\ \sigma_{i j}^{\varepsilon} n_{j}=g_{i} & \text { on } \Gamma_{g}, \\ \sigma_{i j, j}^{\varepsilon}=0 & \text { in } B^{\varepsilon}, \\ {\left[\left[\sigma_{i 3}^{\varepsilon}\right]\right]_{\varepsilon}^{ \pm}=0} & \text { on } S_{ \pm}^{\varepsilon}, \\ {\left[\left[u_{i}^{\varepsilon}\right]\right]_{\varepsilon}^{ \pm}=0} & \text { on } S_{ \pm}^{\varepsilon}, \\ u_{i}^{\varepsilon}=0 & \text { on } \Gamma_{u}, \\ \sigma_{i j}^{\varepsilon}=\left(a_{ \pm}\right)_{i j k l} e_{k l}\left(\mathbf{u}^{\varepsilon}\right) & \text { in } \Omega_{ \pm}^{\varepsilon}, \\ \sigma_{i j}^{\varepsilon}=\left(b^{\varepsilon}\right)_{i j k l} e_{k l}\left(\mathbf{u}^{\varepsilon}\right) & \text { in } B^{\varepsilon},\end{cases}$

with $\mathbf{u}^{\varepsilon}=u_{i}^{\varepsilon} \mathbf{e}_{i}$ a displacement from $\Omega_{+}^{\varepsilon} \cup B^{\varepsilon}$ into $\mathrm{IR}^{3}$, $\boldsymbol{\sigma}^{\varepsilon}=\sigma_{i j}^{\varepsilon} \mathbf{e}_{i} \otimes \mathbf{e}_{j}$ the Cauchy stress, $\mathbf{e}\left(\mathbf{u}^{\varepsilon}\right)$ the strain tensor under the small perturbations hypothesis $\left(e_{i j}=1 / 2\left(u_{i, j}+u_{j, i}\right)\right)$ and $[[f]]_{\varepsilon}^{ \pm}$the jump of $f$ at $S_{ \pm}^{\varepsilon}$. The materials of the adherents and of the thin interphase are assumed to be homogeneous and linearly elastic and $\mathbf{a}_{ \pm}, \mathbf{b}^{\varepsilon}$ are taken to denote the fourth-order elasticity tensors of the adherents and of the interphase, respectively. The tensors $\mathbf{a}_{ \pm}, \mathbf{b}^{\varepsilon}$ are assumed to be symmetric, with the minor and major symmetries, and positive definite. It is well known that the above equilibrium problem admits an unique solution. At least two different methods are possible to study asymptotically the problem (5), by using the minimization of the total energy [18, 19, 42, 43, 45] or, directly, by using the equilibrium equations $[9,16,17,20-23,34]$. In this paper, the last method was preferred.

\subsection{The rescaled equilibrium problem}

Because the thickness of the interphase is very small, it is natural to seek an approximated solution of problem (5) as the small parameter $\varepsilon$ vanishes. In this paper the method of matched asymptotic expansions is applied, which is based on a rescaling of the domains and on series expansions of the (rescaled) relevant fields (displacement and stress vector fields) with respect to $\varepsilon$. In the following, the symbol $(\wedge)$ will indicate the rescaled fields in the adhesive and the symbol (-) the rescaled fields in the adherents.

According to the method of matched asymptotic expansions, a classical change of scale is first applied to the adhesive and to the adherents [6]:

$$
\begin{aligned}
& \hat{\mathbf{p}}:\left(x_{1}, x_{2}, x_{3}\right) \mapsto\left(z_{1}, z_{2}, z_{3}\right)=\left(x_{1}, x_{2}, \frac{x_{3}}{\varepsilon}\right), \\
& \overline{\mathbf{p}}:\left(x_{1}, x_{2}, x_{3}\right) \mapsto\left(z_{1}, z_{2}, z_{3}\right)=\left(x_{1}, x_{2}, x_{3} \pm 1 / 2 \mp \varepsilon / 2\right) .
\end{aligned}
$$

Let $\hat{\mathbf{u}}^{\varepsilon}:=\mathbf{u}^{\varepsilon} \circ \hat{\mathbf{p}}^{-1}$ be a displacement from the rescaled interphase

$B=\left\{\left(x_{1}, x_{2}, x_{3}\right) \in \Omega:-\frac{1}{2}<x_{3}<\frac{1}{2}\right\}$,

and let $\overline{\mathbf{u}}^{\varepsilon}:=\mathbf{u}^{\varepsilon} \circ \overline{\mathbf{p}}^{-1}$ be a displacement from the rescaled adherents

$\Omega_{ \pm}=\left\{\left(x_{1}, x_{2}, x_{3}\right) \in \Omega: \pm x_{3}>\frac{1}{2}\right\}$.

In (7) the plus (minus) sign applied in $\Omega_{+}\left(\Omega_{-}\right)$. Under the change of variables, the domains $\Gamma_{u}$ and $\Gamma_{g}$ are transformed into the domains denoted by $\bar{\Gamma}_{u}$ and $\bar{\Gamma}_{g}$, respectively. The external forces are assumed to be independent of $\varepsilon$, thus it is set $\bar{f}\left(z_{1}, z_{2}, z_{3}\right)=f\left(x_{1}, x_{2}, x_{3}\right), \bar{g}\left(z_{1}, z_{2}, z_{3}\right)=g\left(x_{1}, x_{2}, x_{3}\right)$.

\subsection{Asymptotic expansions}

Within the rescaled configuration (cf. Fig. 1b), the asymptotic expansions of the displacement fields in the adherents and in the interphase take the following form:

$\mathbf{u}^{\varepsilon}\left(z_{1}, z_{2}, z_{3}\right)=\mathbf{u}^{0}+\varepsilon \mathbf{u}^{1}+\varepsilon^{2} \mathbf{u}^{2}+o\left(\varepsilon^{2}\right)$,

$\hat{\mathbf{u}}^{\varepsilon}\left(z_{1}, z_{2}, z_{3}\right)=\hat{\mathbf{u}}^{0}+\varepsilon \hat{\mathbf{u}}^{1}+\varepsilon^{2} \hat{\mathbf{u}}^{2}+o\left(\varepsilon^{2}\right)$,

$\overline{\mathbf{u}}^{\varepsilon}\left(z_{1}, z_{2}, z_{3}\right)=\overline{\mathbf{u}}^{0}+\varepsilon \overline{\mathbf{u}}^{1}+\varepsilon^{2} \overline{\mathbf{u}}^{2}+o\left(\varepsilon^{2}\right)$.

Starting from Eq. (11), the adhesive's strain tensor is obtained:

$\mathbf{e}\left(\hat{\mathbf{u}}^{\varepsilon}\right)=\varepsilon^{-1} \hat{\mathbf{e}}^{-1}+\hat{\mathbf{e}}^{0}+\varepsilon \hat{\mathbf{e}}^{1}+O\left(\varepsilon^{2}\right)$

with:

$\hat{\mathbf{e}}^{-1}:=\left[\begin{array}{cc}0 & \frac{1}{2} \hat{u}_{\alpha, 3}^{0} \\ \frac{1}{2} \hat{u}_{\alpha, 3}^{0} & \hat{u}_{3,3}^{0}\end{array}\right]$

and

$\hat{\mathbf{e}}^{k}:=\left[\begin{array}{cc}\left.\operatorname{Sym}\left(\hat{u}_{\alpha, \beta}^{k}\right)\right) & \frac{1}{2}\left(\hat{u}_{3, \alpha}^{k}+\hat{u}_{\alpha, 3}^{k+1}\right) \\ \frac{1}{2}\left(\hat{u}_{3, \alpha}^{k}+\hat{u}_{\alpha, 3}^{k+1}\right) & \hat{u}_{3,3}^{k+1}\end{array}\right]$,

where $\alpha=1,2, \operatorname{Sym}(\cdot)$ gives the symmetric part of a tensor and $k=0,1, \ldots$

The strain tensor in the adherents can be obtained as:

$\mathbf{e}\left(\overline{\mathbf{u}}^{\varepsilon}\right)=\varepsilon^{-1} \overline{\mathbf{e}}^{-1}+\overline{\mathbf{e}}^{0}+\varepsilon \overline{\mathbf{e}}^{1}+O\left(\varepsilon^{2}\right)$

with:

$\overline{\mathbf{e}}^{-1}=\mathbf{0}$, 
$\overline{\mathbf{e}}^{k}=\left[\begin{array}{cc}\operatorname{Sym}\left(\bar{u}_{\alpha, \beta}^{k}\right) & \frac{1}{2}\left(\bar{u}_{3, \alpha}^{k}+\bar{u}_{\alpha, 3}^{k}\right) \\ \frac{1}{2}\left(\bar{u}_{3, \alpha}^{k}+\bar{u}_{\alpha, 3}^{k}\right) & \bar{u}_{3,3}^{k}\end{array}\right]$,

and again $k=0,1, \ldots$ The stress fields in the rescaled adhesive and adherents, denoted $\hat{\boldsymbol{\sigma}}^{\varepsilon}=\boldsymbol{\sigma} \circ \hat{\mathbf{p}}^{-1}$ and $\overline{\boldsymbol{\sigma}}^{\varepsilon}=\boldsymbol{\sigma} \circ \overline{\mathbf{p}}^{-1}$ respectively, are represented in asymptotic expansions:

$\boldsymbol{\sigma}^{\varepsilon}=\boldsymbol{\sigma}^{0}+\varepsilon \boldsymbol{\sigma}^{1}+O\left(\varepsilon^{2}\right)$,

$\hat{\boldsymbol{\sigma}}^{\varepsilon}=\hat{\boldsymbol{\sigma}}^{0}+\varepsilon \hat{\boldsymbol{\sigma}}^{1}+O\left(\varepsilon^{2}\right)$,

$\overline{\boldsymbol{\sigma}}^{\varepsilon}=\overline{\boldsymbol{\sigma}}^{0}+\varepsilon \overline{\boldsymbol{\sigma}}^{1}+O\left(\varepsilon^{2}\right)$.

As body forces are neglected in the adhesive, the equilibrium equation can be written at the two first orders as:

$\hat{\boldsymbol{\sigma}}_{i 3,3}^{0}=0$,

$\hat{\boldsymbol{\sigma}}_{i 1,1}^{0}+\widehat{\boldsymbol{\sigma}}_{i 2,2}^{0}+\widehat{\boldsymbol{\sigma}}_{i 3,3}^{1}=0$,

where $i=1,2,3$.

Equation (22) shows that the stress vector $\hat{\boldsymbol{\sigma}}_{i 3}^{0}$ is constant with respect to $z_{3}$ in the adhesive, and thus it can be written:

$\left[\hat{\sigma}_{i 3}^{0}\right]=\mathbf{0}$,

where [.] denotes the jump between $z_{3}=\frac{1}{2}$ and $z_{3}=-\frac{1}{2}$.

Analogously, equation (23) can be rewritten in the following integrated form:

$\left[\hat{\boldsymbol{\sigma}}_{i 3}^{1}\right]=-\left[\hat{\boldsymbol{\sigma}}_{i 1,1}^{0}\right]-\left[\hat{\boldsymbol{\sigma}}_{i 2,2}^{0}\right]$.

All equations written so far are general in the sense that they are independent of the constitutive behavior of the materials.

The equilibrium equation in the adherents can be written as:

$\bar{\sigma}_{i j, j}^{0}+f_{i}=0$,

$\bar{\sigma}_{i j, j}^{1}=0$.

As a perfect contact law between the adhesive and the adherents is assumed, the continuity of the displacement and stress vector fields is enforced. In particular, the continuity of the displacements leads to [45]:

$\mathbf{u}^{0}\left(\overline{\mathbf{x}}, 0^{ \pm}\right)=\hat{\mathbf{u}}^{0}\left(\overline{\mathbf{z}}, \pm \frac{1}{2}\right)=\overline{\mathbf{u}}^{0}\left(\overline{\mathbf{z}}, \pm \frac{1}{2}\right)$,

$\mathbf{u}^{1}\left(\overline{\mathbf{x}}, 0^{ \pm}\right) \pm \frac{1}{2} \mathbf{u}_{, 3}^{0}\left(\overline{\mathbf{x}}, 0^{ \pm}\right)=\widehat{\mathbf{u}}^{1}\left(\overline{\mathbf{z}}, \pm \frac{1}{2}\right)=\overline{\mathbf{u}}^{-1}\left(\overline{\mathbf{z}}, \pm \frac{1}{2}\right)$, and the continuity of the stress vector gives the following conditions:

$\boldsymbol{\sigma}_{i 3}^{0}\left(\overline{\mathbf{x}}, 0^{ \pm}\right)=\hat{\boldsymbol{\sigma}}_{i 3}^{0}\left(\overline{\mathbf{z}}, \pm \frac{1}{2}\right)=\overline{\boldsymbol{\sigma}}_{i 3}^{0}\left(\overline{\mathbf{z}}, \pm \frac{1}{2}\right)$

$\boldsymbol{\sigma}_{i 3}^{1}\left(\overline{\mathbf{x}}, 0^{ \pm}\right) \pm \frac{1}{2} \boldsymbol{\sigma}_{i 3,3}^{0}\left(\overline{\mathbf{x}}, 0^{ \pm}\right)=\hat{\boldsymbol{\sigma}}_{i 3}^{1}\left(\overline{\mathbf{z}}, \pm \frac{1}{2}\right)=\overline{\boldsymbol{\sigma}}_{i 3}^{1}\left(\overline{\mathbf{z}}, \pm \frac{1}{2}\right)$

for $i=1,2,3$

Following [45], the matrices $\mathbf{K}_{\varepsilon}^{j l}$ (with $j, l=1,2,3$ ) are introduced, whose components are defined by the relation:

$\left(K_{\varepsilon}^{j l}\right)_{k i}:=b_{i j k l}^{\varepsilon}$.

In view of the symmetry properties of the elasticity tensor $\mathbf{b}^{\varepsilon}$, it results that $\mathbf{K}_{\varepsilon}^{j l}=\left(\mathbf{K}_{\varepsilon}^{l j}\right)^{T}$, with $j, l=1,2,3$.

\subsection{Internal/interphase analysis}

In the following, two specific cases of linearly elastic material are studied for the interphase. One, called "soft" material, is characterized by elastic moduli which are linearly rescaled with respect to the thickness $\varepsilon$; the second case, called "hard" material, is characterized by elastic moduli independent of the thickness $\varepsilon$.

\subsubsection{Soft interphase analysis}

Assuming that the interphase is "soft", one defines:

$\mathbf{b}^{\varepsilon}=\varepsilon \mathbf{b}$,

where the tensor $\mathbf{b}$ does not depend on $\varepsilon$. Accordingly to position (32), it is set:

$K_{k i}^{j l}:=b_{i j k l}$.

Introducing the expansions in the constitutive equations leads to:

$\hat{\boldsymbol{\sigma}}^{0}+\varepsilon \hat{\boldsymbol{\sigma}}^{1}=\mathbf{b}\left(\hat{\mathbf{e}}^{-1}+\varepsilon \hat{\mathbf{e}}^{0}\right)+o(\varepsilon)$

$\hat{\boldsymbol{\sigma}}^{0}=b\left(\hat{\mathbf{e}}^{-1}\right)$

$\hat{\boldsymbol{\sigma}}^{1}=b\left(\hat{\mathbf{e}}^{0}\right)$

and

$\hat{\boldsymbol{\sigma}}^{0} \mathbf{i}_{j}=\mathbf{K}^{3 j} \hat{\mathbf{u}}_{, 3}^{0}$

for $j=1,2,3$, and

$\hat{\boldsymbol{\sigma}}^{0} \mathbf{i}_{3}=\mathbf{K}^{33}\left[\hat{\mathbf{u}}^{0}\right]$,

which represents the classical law for a soft interface.

Analogously, it is obtained:

$\left[\hat{\boldsymbol{\sigma}}^{1} \mathbf{i}_{3}\right]=-\mathbf{K}^{31}\left[\hat{\mathbf{u}}^{0}\right]_{, 1}-\mathbf{K}^{32}\left[\hat{\mathbf{u}}^{0}\right]_{, 2}$ 
Denoting $<f>(\overline{\mathbf{z}}):=\frac{1}{2}\left(f\left(\overline{\mathbf{z}}, \frac{1}{2}\right)+f\left(\overline{\mathbf{z}},-\frac{1}{2}\right)\right)$, it is obtained:

$\left[\hat{\mathbf{u}}^{1}\right]=\left(\mathbf{K}^{33}\right)^{-1}\left(<\hat{\boldsymbol{\sigma}}^{1} \mathbf{i}_{3}>-\mathbf{K}^{\alpha 3}<\hat{\mathbf{u}}^{0}>_{, \alpha}\right)$

where the sum over $\alpha=1,2$ is performed.

The complete problem for soft interface can be written at the two first orders as:

$\begin{cases}\sigma_{i j, j}^{0}+f_{i}=0 & \text { in } \Omega_{ \pm}, \\ \sigma_{i j}^{0} n_{j}=g_{i} & \text { on } \Gamma_{g}, \\ u_{i}^{0}=0 & \text { on } \Gamma_{u}, \\ \sigma_{i j}^{0}=\left(a_{ \pm}\right)_{i j k l} e_{k l}\left(\mathbf{u}^{0}\right) & \text { in } \Omega_{ \pm} \\ {\left[\boldsymbol{\sigma}_{i 3}^{0}\right]=\mathbf{0}} & \text { on } S, \\ \boldsymbol{\sigma}_{i 3}^{0}=\mathbf{K}_{i k}^{33}\left[u_{k}^{0}\right] & \text { on } S .\end{cases}$

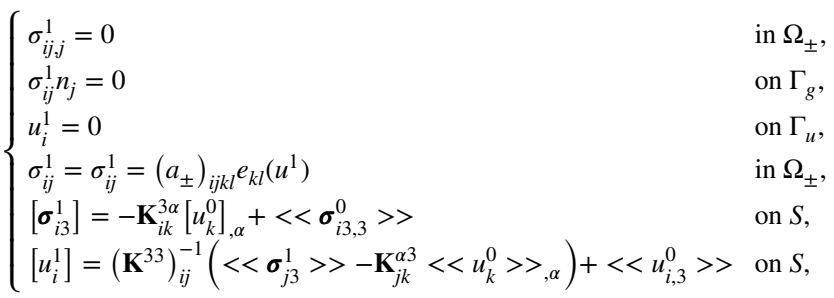

where $<<f>>(\overline{\mathbf{x}}):=\frac{1}{2}\left(f\left(\overline{\mathbf{x}}, 0^{+}\right)+f\left(\overline{\mathbf{x}}, 0^{-}\right)\right)$.
Taking into account the first equation in (45) and using the positive definiteness of the tensor $\mathbf{b}$, one obtains:

$\hat{\mathbf{u}}_{3}^{0}=0 \Rightarrow\left[\hat{\mathbf{u}}^{0}\right]=\mathbf{0}$,

which corresponds to the kinematics of the perfect interface.

Analogously, it is obtained:

$\left[\hat{\mathbf{u}}^{1}\right]=\left(\mathbf{K}^{33}\right)^{-1}\left(\hat{\boldsymbol{\sigma}}^{0} \mathbf{i}_{3}-\mathbf{K}^{\alpha 3} \hat{\mathbf{u}}_{, \alpha}^{0}\right)$,

and

$\left[\hat{\boldsymbol{\sigma}}^{1} \mathbf{i}_{3}\right]=\left(-\mathbf{K}^{\alpha \beta} \hat{\mathbf{u}}_{, \beta}^{0}-\mathbf{K}^{3 \alpha}\left[\hat{\mathbf{u}}^{1}\right]\right)_{, \alpha}$

$=\left(-\mathbf{K}^{\alpha \beta} \hat{\mathbf{u}}_{, \beta}^{0}-\mathbf{K}^{3 \alpha}\left(\mathbf{K}^{33}\right)^{-1}\left(\hat{\boldsymbol{\sigma}}^{0} \mathbf{i}_{3}-\mathbf{K}^{\beta 3} \hat{\mathbf{u}}_{, \beta}^{0}\right)\right)_{, \alpha}$.

The complete problem for hard interface can be written at the two first orders as:

$\begin{cases}\sigma_{i j . j}^{0}+f_{i}=0 & \text { in } \Omega_{ \pm}, \\ \sigma_{i j}^{0} n_{j}=g_{i} & \text { on } \Gamma_{g}, \\ u_{i}^{0}=0 & \text { on } \Gamma_{u}, \\ \sigma_{i j}^{0}=\left(a_{ \pm}\right)_{i j k l} e_{k l}\left(u^{0}\right) & \text { in } \Omega_{ \pm}, \\ {\left[\boldsymbol{\sigma}_{i j}^{0}\right]=\mathbf{0}} & \text { on } S \\ {\left[u_{i}^{0}\right]=0} & \text { on } S\end{cases}$

in $\Omega_{ \pm}$,

on $\Gamma_{g}$,

on $\Gamma_{u}$,

in $\Omega_{ \pm}$,

on $S$,

on $S$. $\mathbf{b}^{\varepsilon}=\mathbf{b}$,

where the tensor $\mathbf{b}$ is independent of $\varepsilon$, and $\mathbf{K}^{j l}$ is still taken to denote the matrices such that $K_{k i}^{j l}:=b_{i j k l}$.

Taking into account the relations (13) and (20), the stress-strain relationship takes the following form:

$\hat{\boldsymbol{\sigma}}^{0}+\varepsilon \hat{\boldsymbol{\sigma}}^{1}=\mathbf{b}\left(\varepsilon^{-1} \hat{\mathbf{e}}^{-1}+\hat{\mathbf{e}}^{0}+\varepsilon \hat{\mathbf{e}}^{1}\right)+o(\varepsilon)$,

which gives

$\mathbf{0}=\mathbf{b}\left(\hat{\mathbf{e}}^{-1}\right)$,

$\hat{\boldsymbol{\sigma}}^{0}=\mathbf{b}\left(\hat{\mathbf{e}}^{0}\right)$.

\section{How introduce roughness?}

\subsection{The three-dimensional equilibrium problem}

In this Section, a general methodology is proposed to take into account the interphase roughness. In particular, two given positive-valued dimensionless roughness functions, $\eta^{ \pm} \in C^{0}\left(S, \mathrm{IR}^{2}\right)$, describing a smooth (i.e., $\eta^{ \pm}$are independent of $\varepsilon$ ) surface roughness between the adherents and the interphase, are introduced. The domains $B^{\varepsilon}, \Omega_{ \pm}^{\varepsilon}$ and $S_{ \pm}^{\varepsilon}$ are then defined as: 
$B^{\varepsilon}=\left\{\left(x_{1}, x_{2}, x_{3}\right) \in \Omega:-\frac{\varepsilon}{2} \eta^{-}\left(x_{1}, x_{2}\right)<x_{3}<\frac{\varepsilon}{2} \eta^{+}\left(x_{1}, x_{2}\right)\right\}$,

$\Omega_{ \pm}^{\varepsilon}=\left\{\left(x_{1}, x_{2}, x_{3}\right) \in \Omega: \pm x_{3}>\frac{\varepsilon}{2} \eta^{ \pm}\left(x_{1}, x_{2}\right)\right\}$,

and the interfaces between the adherents and the adhesive interphase are

$S_{ \pm}^{\varepsilon}=\left\{\left(x_{1}, x_{2}, x_{3}\right) \in \Omega: x_{3}= \pm \frac{\varepsilon}{2} \eta^{ \pm}\left(x_{1}, x_{2}\right)\right\}$.

We introduce two modified changes of variables:

$\hat{\mathbf{p}}^{\eta}:\left(x_{1}, x_{2}, x_{3}\right) \mapsto\left(z_{1}, z_{2}, z_{3}\right)=\left(x_{1}, x_{2}, \frac{x_{3}}{\eta^{+} \varepsilon}\right), x_{3} \geq 0$,

$\hat{\mathbf{p}}^{\eta}:\left(x_{1}, x_{2}, x_{3}\right) \mapsto\left(z_{1}, z_{2}, z_{3}\right)=\left(x_{1}, x_{2}, \frac{x_{3}}{\eta^{-} \varepsilon}\right), x_{3} \leq 0$,

$\overline{\mathbf{p}}^{\eta}:\left(x_{1}, x_{2}, x_{3}\right) \mapsto\left(z_{1}, z_{2}, z_{3}\right)=\left(x_{1}, x_{2}, x_{3} \pm 1 / 2-\eta^{+} \varepsilon / 2\right), x_{3} \geq 0$,

$\overline{\mathbf{p}}^{\eta}:\left(x_{1}, x_{2}, x_{3}\right) \mapsto\left(z_{1}, z_{2}, z_{3}\right)=\left(x_{1}, x_{2}, x_{3} \pm 1 / 2+\eta^{-} \varepsilon / 2\right), x_{3} \leq 0$.

Note that with these changes of variables, in the adhesive one has $d x_{3}=\eta^{ \pm} d z_{3}$.

As a perfect contact law between the adhesive and the adherents is assumed, the continuity of the displacement and stress vector fields is enforced. In particular, the continuity of the displacements gives:

$\mathbf{u}^{\varepsilon}\left(\overline{\mathbf{x}}, \pm \frac{\eta^{ \pm} \varepsilon}{2}\right)=\hat{\mathbf{u}}^{\varepsilon}\left(\overline{\mathbf{z}}, \pm \frac{1}{2}\right)=\overline{\mathbf{u}}^{\varepsilon}\left(\overline{\mathbf{z}}, \pm \frac{1}{2}\right)$

Expanding the displacement in the adherent, $\mathbf{u}^{\varepsilon}$, in Taylor series along the $x_{3}$-direction and taking into account the asymptotic expansion, it results:

$$
\begin{aligned}
\mathbf{u}^{\varepsilon}\left(\overline{\mathbf{x}}, \pm \frac{\eta^{ \pm} \varepsilon}{2}\right) & =\mathbf{u}^{\varepsilon}\left(\overline{\mathbf{x}}, 0^{ \pm}\right) \pm \frac{\eta^{ \pm} \varepsilon}{2} \mathbf{u}_{, 3}^{\varepsilon}\left(\overline{\mathbf{x}}, 0^{ \pm}\right)+\cdots \\
& =\mathbf{u}^{0}\left(\overline{\mathbf{x}}, 0^{ \pm}\right)+\varepsilon \mathbf{u}^{1}\left(\overline{\mathbf{x}}, 0^{ \pm}\right) \pm \frac{\eta^{ \pm} \varepsilon}{2} \mathbf{u}_{, 3}^{0}\left(\overline{\mathbf{x}}, 0^{ \pm}\right)+\cdots
\end{aligned}
$$

Thus it is obtained:

$$
\begin{aligned}
\mathbf{u}^{0}\left(\overline{\mathbf{x}}, 0^{ \pm}\right)+\varepsilon \mathbf{u}^{1}\left(\overline{\mathbf{x}}, 0^{ \pm}\right) \pm \frac{\eta^{ \pm} \varepsilon}{2} \mathbf{u}_{3,}^{0}\left(\overline{\mathbf{x}}, 0^{ \pm}\right)+\cdots & =\hat{\mathbf{u}}^{0}\left(\overline{\mathbf{z}}, \pm \frac{1}{2}\right)+\varepsilon \hat{\mathbf{u}}^{1}\left(\overline{\mathbf{z}}, \pm \frac{1}{2}\right)+\cdots \\
& =\overline{\mathbf{u}}^{0}\left(\overline{\mathbf{z}}, \pm \frac{1}{2}\right)+\varepsilon \overline{\mathbf{u}}^{1}\left(\overline{\mathbf{z}}, \pm \frac{1}{2}\right)+\cdots
\end{aligned}
$$

Identifying the terms with the same powers of $\varepsilon$, Eq. (61) gives:

$\mathbf{u}^{0}\left(\overline{\mathbf{x}}, 0^{ \pm}\right)=\hat{\mathbf{u}}^{0}\left(\overline{\mathbf{z}}, \pm \frac{1}{2}\right)=\overline{\mathbf{u}}^{0}\left(\overline{\mathbf{z}}, \pm \frac{1}{2}\right)$ $\mathbf{u}^{1}\left(\overline{\mathbf{x}}, 0^{ \pm}\right) \pm \frac{1}{2} \eta^{ \pm} \mathbf{u}_{, 3}^{0}\left(\overline{\mathbf{x}}, 0^{ \pm}\right)=\widehat{\mathbf{u}}^{1}\left(\overline{\mathbf{z}}, \pm \frac{1}{2}\right)=\overline{\mathbf{u}}^{1}\left(\overline{\mathbf{z}}, \pm \frac{1}{2}\right)$

Following a similar analysis for the stress vector, analogous results are obtained:

$\boldsymbol{\sigma}_{i 3}^{0}\left(\overline{\mathbf{x}}, 0^{ \pm}\right)=\hat{\boldsymbol{\sigma}}_{i 3}^{0}\left(\overline{\mathbf{z}}, \pm \frac{1}{2}\right)=\overline{\boldsymbol{\sigma}}_{i 3}^{0}\left(\overline{\mathbf{z}}, \pm \frac{1}{2}\right)$

$\boldsymbol{\sigma}_{i 3}^{1}\left(\overline{\mathbf{x}}, 0^{ \pm}\right) \pm \frac{1}{2} \eta^{ \pm} \boldsymbol{\sigma}_{i 3,3}^{0}\left(\overline{\mathbf{x}}, 0^{ \pm}\right)=\hat{\boldsymbol{\sigma}}_{i 3}^{1}\left(\overline{\mathbf{z}}, \pm \frac{1}{2}\right)=\overline{\boldsymbol{\sigma}}_{i 3}^{1}\left(\overline{\mathbf{z}}, \pm \frac{1}{2}\right)$,

for $i=1,2,3$. Note that using this technique, the jump the stress vector at order zero is not modified. However, taking the derivatives along the third axis shows that the matching conditions at order one are modified as follows:

$\left[\mathbf{u}^{1}\right](\overline{\mathbf{x}})=\left[\hat{\mathbf{u}}^{1}\right](\overline{\mathbf{x}})+<<<\mathbf{u}_{, 3}^{0}>>>(\overline{\mathbf{x}})$,

$\left[\boldsymbol{\sigma}^{1} \mathbf{e}_{3}\right](\overline{\mathbf{x}})=\left[\hat{\boldsymbol{\sigma}}^{1} \mathbf{e}_{3}\right](\overline{\mathbf{x}})+<<<\boldsymbol{\sigma}_{, 3}^{0} \mathbf{e}_{3}>>>(\overline{\mathbf{x}})$,

with $<<<f>>>(\cdot)=1 / 2 \eta^{+} f\left(\cdot, 0^{+}\right)+1 / 2 \eta^{-} f\left(\cdot, 0^{-}\right)$.

Now the equilibrium equation at order zero gives:

$\frac{1}{\eta^{ \pm}} \hat{\boldsymbol{\sigma}}_{i 3,3}^{0}=\mathbf{0}$

and thus,

$\left[\hat{\boldsymbol{\sigma}}_{i 3}^{0}\right]=0$.

At order one, the equilibrium equation leads to the condition

$\hat{\boldsymbol{\sigma}}_{i 3,3}^{1}=-\eta^{ \pm} \hat{\boldsymbol{\sigma}}_{i \alpha, \alpha}^{0}$

\subsection{Soft interphase analysis}

In this section, the interphase is assumed to be "soft". The notation are the same as in Sect. 2. It is obvious to observe that Eq. (37) becomes

$\eta^{ \pm} \hat{\boldsymbol{\sigma}}^{0} \mathbf{i}_{j}=\mathbf{K}^{3 j} \hat{\mathbf{u}}_{, 3}^{0}$

for $j=1,2,3$, and

$\hat{\boldsymbol{\sigma}}^{0} \mathbf{i}_{3}=\frac{1}{\eta} \mathbf{K}^{33}\left[\hat{\mathbf{u}}^{0}\right]$

where $\eta=\frac{\eta^{+}+\eta^{-}}{2}$. Equation (72) describes an imperfect interface mode taking into account roughness.

Combining Eqs. (70) and (71), it is obtained: 


$$
\left[\hat{\boldsymbol{\sigma}}^{1} \mathbf{i}_{3}\right]=-\mathbf{K}^{31}\left[\hat{\mathbf{u}}^{0}\right]_{, 1}-\mathbf{K}^{32}\left[\hat{\mathbf{u}}^{0}\right]_{, 2}
$$

The constitutive equation gives:

$$
\hat{\boldsymbol{\sigma}}^{1} \mathbf{i}_{j}=\mathbf{K}^{\alpha j} \hat{\mathbf{u}}_{, \alpha}^{0}+\frac{1}{\eta^{ \pm}} \mathbf{K}^{3 j} \hat{\mathbf{u}}_{, 3}^{0}
$$
(74)]:

Being $\hat{\boldsymbol{\sigma}}^{1}$ and $\hat{\mathbf{u}}_{, \alpha}^{0}$ linear in $z_{3}$, we have [with $j=3$ in Eq.

$$
\left[\hat{\mathbf{u}}^{1}\right]=\eta\left(\mathbf{K}^{33}\right)^{-1}\left(<\hat{\boldsymbol{\sigma}}^{1} \mathbf{i}_{3}>-\mathbf{K}^{\alpha 3}<\hat{\mathbf{u}}^{0}>_{, \alpha}\right)
$$

The complete problem for soft interface can be written at the two first orders as:

$$
\begin{cases}\sigma_{i j, j}^{0}+f_{i}=0 & \text { in } \Omega_{ \pm} \\ \sigma_{i j}^{0} n_{j}=g_{i} & \text { on } \Gamma_{g} \\ u_{i}^{0}=0 & \text { on } \Gamma_{u} \\ \sigma_{i j}^{0}=\left(a_{ \pm}\right)_{i j k l} e_{k l}\left(\mathbf{u}^{0}\right) & \text { in } \Omega_{ \pm} \\ {\left[\boldsymbol{\sigma}_{i 3}^{0}\right]=\mathbf{0}} & \text { on } S \\ \boldsymbol{\sigma}_{i 3}^{0}=\frac{1}{\eta} \mathbf{K}_{i k}^{33}\left[u_{k}^{0}\right] & \text { on } S\end{cases}
$$

$$
\begin{cases}\sigma_{i j, j}^{1}=0 & \text { in } \Omega_{ \pm}, \\ \sigma_{i j}^{1} n_{j}=0 & \text { on } \Gamma_{g}, \\ u_{i}^{1}=0 & \text { on } \Gamma_{u}, \\ \sigma_{i j}^{1}=\left(a_{ \pm}\right)_{i j k l} e_{k l}\left(u^{1}\right) & \text { in } \Omega_{ \pm}, \\ {\left[\boldsymbol{\sigma}_{i 3}^{1}\right]=\eta\left(K_{i j}^{\alpha \beta} u_{j, \beta}^{0}+\left(\mathbf{K}^{3 \alpha}\left(\mathbf{K}^{33}\right)^{-1}\right)_{i j}\left(\hat{\boldsymbol{\sigma}}_{j 3}^{0}-\mathbf{K}_{j k}^{\beta 3} u_{k, \beta}^{0}\right)\right)_{, \alpha}+<<<\boldsymbol{\sigma}_{i 3,3}^{0}>>>} & \text { on } S, \\ {\left[u_{i}^{1}\right]=\eta\left(\mathbf{K}^{33}\right)_{i j}^{-1}\left(\boldsymbol{\sigma}_{j 3}^{0}-\mathbf{K}_{j k}^{\alpha 3} u_{k, \alpha}^{0}\right)+<<<u_{i, 3}^{0}>>>} & \text { on } S .\end{cases}
$$

$$
\begin{cases}\sigma_{i j, j}^{1}=0 & \text { in } \Omega_{ \pm}, \\ \sigma_{i j}^{1} n_{j}=0 & \text { on } \Gamma_{g}, \\ u_{i}^{1}=0 & \text { on } \Gamma_{u}, \\ \sigma_{i j}^{1}=\left(\mathbf{a}_{ \pm}\right)_{i j k l} e_{k l}\left(u^{1}\right) & \text { in } \Omega_{ \pm}, \\ {\left[\boldsymbol{\sigma}_{i 3}^{1}\right]=-\mathbf{K}_{i k}^{3 \alpha}\left[u_{k}^{0}\right]_{\alpha}+<<<\boldsymbol{\sigma}_{i 3,3}^{0}>>>} & \text { on } S, \\ {\left[u_{i}^{1}\right]=\eta\left(\mathbf{K}^{33}\right)_{i j}^{-1}\left(<<\boldsymbol{\sigma}_{j 3}^{1}>>-\mathbf{K}_{j k}^{\alpha 3}<<u_{k}^{0}>>{ }_{, \alpha}\right)+<<<u_{i, 3}^{0}>>>} & \text { on } S .\end{cases}
$$

\subsection{Hard interphase analysis}

In this section, a "hard" interphase is considered. The jump in the displacements at order zero is not modified:

$\hat{\mathbf{u}}_{, 3}^{0}=\mathbf{0} \Rightarrow\left[\hat{\mathbf{u}}^{0}\right]=\mathbf{0}$,

giving again the kinematics of the perfect interface.
The integration of the constitutive equations gives:

$\left[\hat{\mathbf{u}}^{1}\right]=\eta\left(\mathbf{K}^{33}\right)^{-1}\left(\hat{\boldsymbol{\sigma}}^{0} \mathbf{i}_{3}-\mathbf{K}^{\alpha 3} \hat{\mathbf{u}}_{, \alpha}^{0}\right)$

and by combining with the equilibrium equation one obtains

$$
\begin{aligned}
& {\left[\hat{\boldsymbol{\sigma}}^{1} \mathbf{i}_{3}\right]=\left(-\mathbf{K}^{\alpha \beta} \hat{\mathbf{u}}_{, \beta}^{0}-\mathbf{K}^{3 \alpha}\left[\hat{\mathbf{u}}^{1}\right]\right)_{, \alpha}} \\
& =-\eta\left(\mathbf{K}^{\alpha \beta} \hat{\mathbf{u}}_{, \beta}^{0}+\mathbf{K}^{\alpha 3}\left(\mathbf{K}^{33}\right)^{-1}\left(\hat{\boldsymbol{\sigma}}^{0} \mathbf{i}_{3}-\mathbf{K}^{\alpha 3} \hat{\mathbf{u}}_{, \alpha}^{0}\right)\right)_{, \alpha}
\end{aligned}
$$

The complete problem for hard interface can be written at the two first orders as:

$$
\begin{cases}\sigma_{i j, j}^{0}+f_{i}=0 & \text { in } \Omega_{ \pm}, \\ \sigma_{i j}^{0} n_{j}=g_{i} & \text { on } \Gamma_{g}, \\ u_{i}^{0}=0 & \text { on } \Gamma_{u}, \\ \sigma_{i j}^{0}=\left(a_{ \pm}\right)_{i j k l} e_{k l}\left(\mathbf{u}^{0}\right) & \text { in } \Omega_{ \pm}, \\ {\left[\boldsymbol{\sigma}_{i 3}^{0}\right]=\mathbf{0}} & \text { on } S, \\ {\left[u_{i}^{0}\right]=0} & \text { on } S .\end{cases}
$$

\section{How introduce microcracks?}

A very simple technique is now proposed to account for the presence of microcracks in the interphase. The idea is to introduce these microcracks (or porosity) directly in the elastic constitutive equations. A heterogeneous material is considered made of an elastic material with cracks. To model the macroscopic behavior of cracked materials, homogenization models can be used and then a new homogeneous equivalent medium can be defined. The stiffness tensor depends on the crack length and orientation. In [11, 40, 41], the authors introduce cracks by applying the methodology developed by Kachanov and his collaborators [30, 48]. In this approach, a crack density $\rho$ is introduced. In two dimensions, this density is seen as the ratio between the square of the length of the crack $l$ and the elementary volume describing the material at the microscale. Thus, the 
Fig. 2 Homogenization scheme for micro-cracked interphase media. The homogenized interphase is then treated by the asymptotic method in order to obtain the stiffnesses matrix $\mathbf{K}^{33}$ of the imperfect-interface law
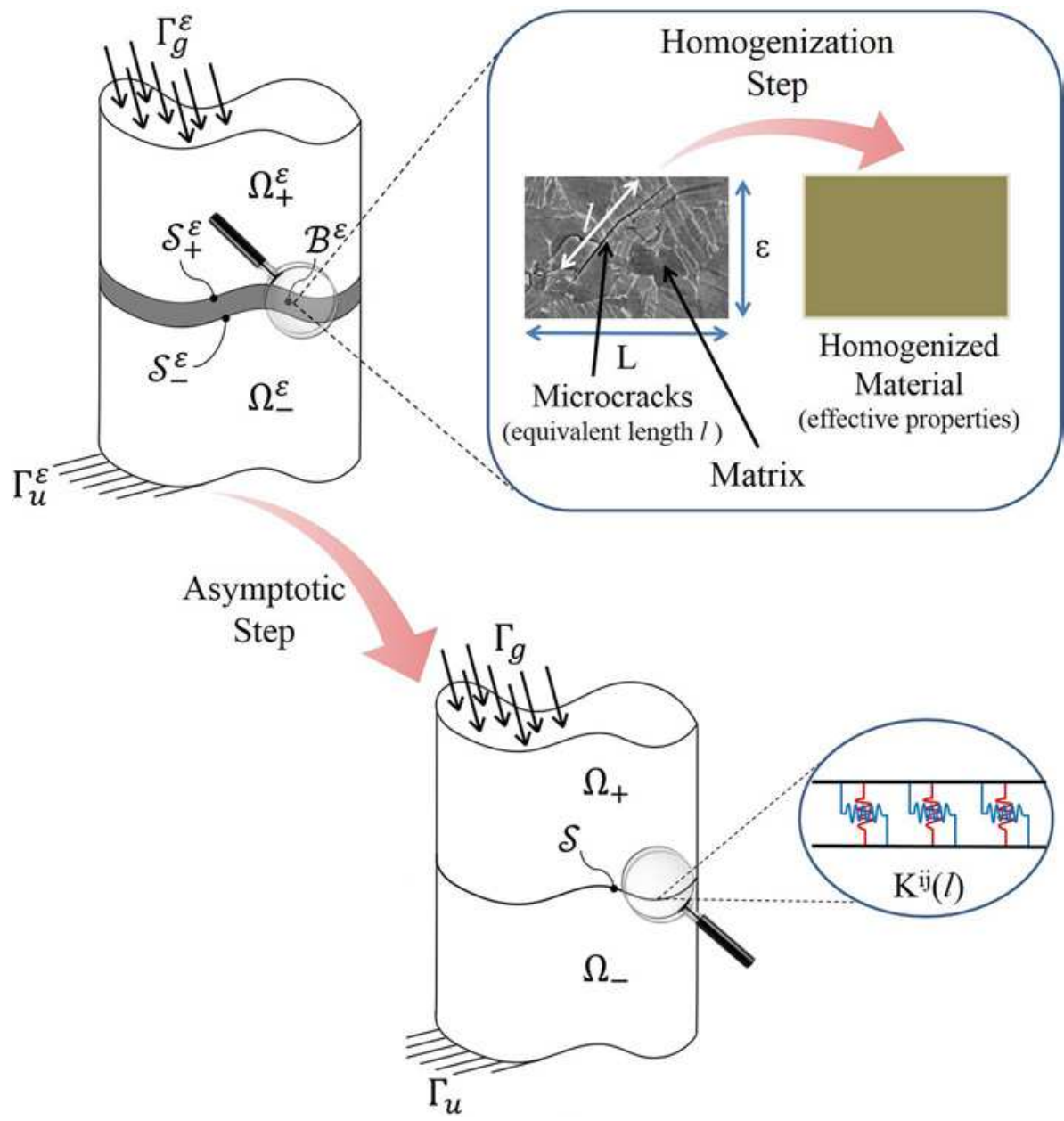

crack density is inversely proportional to the thickness of the interface. The stiffness depends directly on the length $l$. Due this dependence, the next step consists in the asymptotic study of the stiffness coefficients of the homogenized elastic tensor. Obviously, two cases can appear, in the first one, the material is soft, in the second one the material is hard. In the previous sections, it has been shown how it is possible from this starting point to obtain imperfect soft or hard interface models. A scheme of this methodology is proposed in Fig. 2.

Within this section, two examples of homogenization techniques are given. The first one is based on the theory proposed by Kachanov et al. [30, 48]. The results presented here are given for a damaged isotropic material. We take $E^{0}$ ,$\mu^{0}$ and $v^{0}$ to denote the Young's modulus, the shear modulus and the Poisson's ratio of the undamaged material. We assume that the crack lies along the direction of $\mathbf{e}_{1}$. In this case and in two dimensions, the stiffness coefficients of the cracked material, indexed by 1 , are given by
$\left\{\begin{array}{l}E_{1}^{1}=E^{0}, \\ E_{2}^{1}=E^{0}\left(1+\frac{16\left(1-\left(\nu^{0}\right)^{2}\right)}{3} \rho\right)^{-1}, \\ \mu_{12}^{1}=\mu^{0}\left(1+\frac{8\left(1-\nu^{0}\right)}{3\left(1-\nu^{0} / 2\right)} \rho\right)^{-1}, \\ \nu_{12}^{1}=\nu^{0} .\end{array}\right.$

where $\rho=l^{2} / \varepsilon L$ and $L$ is the length of the interface. The subindex $i$ indicates the direction parallel to the crack, i.e. 1 , or orthogonal to the crack, i.e. 2 .

A simple asymptotic study of these coefficients leads to

$\left\{\begin{array}{r}\mathbf{b}^{\varepsilon} \approx \varepsilon \mathbf{b} \\ \mathbf{b}=\mathbf{b}(l)\end{array}\right.$

where $\mathbf{b}^{\varepsilon}$ is effective elastic tensor of the homogenized material. Thus, this homogenization theory leads to a soft material. By considering the microcracks density, the stiffness coefficients are given by 


$$
\left\{\begin{aligned}
E_{1}^{1} & =E^{0}, \\
E_{2}^{1} & \approx \frac{3 \varepsilon E_{0} L}{16 l^{2}\left(1-\left(\nu^{0}\right)^{2}\right)} \\
\mu_{12}^{1} & \approx \frac{3 \varepsilon L \mu^{0}\left(1-\nu^{0}\right)}{16 l^{2}\left(1-\left(\nu^{0}\right)^{2}\right)} \\
\nu_{12}^{1} & =\nu^{0}
\end{aligned}\right.
$$

Note also that, in two dimensions, the normal stiffness and the tangential stiffness take the form

$$
\left\{\begin{array}{l}
K_{N}=\frac{3 E^{0} L}{16 l^{2}\left(1-\left(\nu^{0}\right)^{2}\right)}, \\
K_{T}=\frac{3 E^{0} L\left(v^{0}-2\right)}{32 l^{2}\left(\left(\nu^{0}\right)^{2}-1\right)} .
\end{array}\right.
$$

In the second example of cracked material, we introduce a dilute homogenization scheme or a dilute estimate [4]. Note that other effective coefficients can be found in the literature (see [35] for example). In the case of a dilute estimate, the stiffness coefficients are given by:

$$
\left\{\begin{aligned}
C_{\rho} & =\frac{3(2 v-1)+16\left((1-v)^{2}\right) \rho}{3(2 v-1)+32 v^{2}(1-v) \rho} \\
E_{1}^{1} & =C_{\rho} E^{0} \\
E_{2}^{1} & =E^{0} \\
\mu_{12}^{1} & =C_{\rho} \mu^{0} \\
v_{21}^{1} & =C_{\rho} \nu^{0} .
\end{aligned}\right.
$$

A simple asymptotic study of these coefficients leads to:

$$
\left\{\begin{aligned}
\mathbf{b}^{\varepsilon} & \approx \mathbf{b} \\
\mathbf{b} & =\mathbf{b}(l)
\end{aligned}\right.
$$

These equations thus, lead to a model of hard interface, as defined above. In Sect. 6, these models will be compared within simple numerical examples.

\section{A nonlinear model of imperfect interface accounting for roughness}

In this section, the asymptotic behavior of a joint with an adhesive of vanishing thickness and comprising an isotropic Saint Venant-Kirchhoff material is reviewed. The complete problem is addressed in [44], where the cases of "soft", "hard" and "rigid" adhesive are distinguished, depending upon the rescaling of the adhesive moduli with its thickness. Attention here is restricted to the case of a "hard" adhesive, for which it is intended that its elastic moduli are independent of the adhesive thickness. The roughness of the adhesive is taken into account as in Sect. 3 via the definition of a roughness function [11, 40, 41].

\subsection{The three-dimensional equilibrium problem}

A thin interphase, $B^{\varepsilon}$, joining two adherents, $\Omega_{+}^{\varepsilon}$, is considered and the same notation and assumptions of Sects. 2 and 3 is adopted. The materials of the adherents and of the thin interphase are now modeled as Saint Venant-Kirchhoff isotropic materials, with Lamé's coefficients equal to $\lambda^{ \pm}, \mu^{ \pm}$ in the adherents and $\lambda, \mu$ in the interphase. The latter are assumed to be independent of $\varepsilon$, thus providing a model of "hard" interface, accordingly to the terminology introduced in the previous sections.

The equations governing the equilibrium problem of the composite structure are written as follows:

$\begin{cases}P_{i j, j}^{\varepsilon}+f_{i}=0 & \text { in } \Omega_{ \pm}^{\varepsilon}, \\ P_{i j}^{\varepsilon} n_{j}=g_{i} & \text { on } \Gamma_{g}, \\ P_{i j, j}^{\varepsilon}=0 & \text { in } B^{\varepsilon}, \\ {\left[\left[\mathbf{P}_{i j}^{\varepsilon}\right]\right]_{\varepsilon}^{ \pm}=\mathbf{0}} & \text { on } S_{ \pm}^{\varepsilon}, \\ {\left[\left[u_{i}^{\varepsilon}\right]\right]_{\varepsilon}^{ \pm}=0} & \text { on } S_{ \pm}^{\varepsilon}, \\ u_{i}^{\varepsilon}=0 & \text { on } \Gamma_{u}, \\ P_{i j}^{\varepsilon}=\left(\delta_{i k}+u_{i, k}\right)\left(2 \mu^{ \pm}\left(u_{k, j}+u_{j, k}+\frac{1}{2} u_{h, k} u_{h, j}\right)+\lambda^{ \pm}\left(u_{l, l}+\frac{1}{2}\left|\mathbf{u}_{l}\right|^{2}\right) \delta_{k j}\right) & \text { in } \Omega_{ \pm}^{\varepsilon}, \\ P_{i j}^{\varepsilon}=\left(\delta_{i k}+u_{i, k}\right)\left(2 \mu\left(u_{k, j}+u_{j, k}+\frac{1}{2} u_{h, k} u_{h, j}\right)+\lambda\left(u_{l, l}+\frac{1}{2}\left|\mathbf{u}_{l}\right|^{2}\right) \delta_{k j}\right) & \text { in } B^{\varepsilon},\end{cases}$

with $\mathbf{u}^{\varepsilon}=u_{i}^{\varepsilon} \mathbf{e}_{i}$ a displacement from $\Omega_{ \pm}^{\varepsilon} \cup B^{\varepsilon}$ into $\mathrm{IR}^{3}$, $\mathbf{P}^{\varepsilon}=P_{i j}^{\varepsilon} \mathbf{e}_{i} \otimes \mathbf{e}_{j}$ the corresponding first ${ }^{ \pm}$Piola-Kirchhoff stress and $\delta_{i k}$ the Kronecker symbol.

It is well known that the Saint-Venant Kirchhoff energy is not rank-one convex and thus the direct method of the calculus of variations can not be applied to provide the existence of (weak) solutions of the above equilibrium problem [7, 24]. Existence results are known for special cases, i.e. when the pure displacement boundary value condition is considered and body forces are sufficiently small [10, 27, 29].

\subsection{Asymptotic analysis}

According to the method of matched asymptotic expansions implemented in the present paper, a change of scale is first applied to the adhesive interphase and the adherents. To take into account the roughness of the interphase, we introduce the modified change of variables as in (56-58). Next, the following asymptotic series in the rescaled interphase and adherents domains are introduced:

$\hat{P}_{i j}^{\varepsilon}=\hat{P}_{i j}^{0}+\varepsilon \hat{P}_{i j}^{1}+o(\varepsilon)$,

$\hat{u}_{i}^{\varepsilon}=\hat{u}_{i}^{0}+\varepsilon \hat{u}_{i}^{1}+o(\varepsilon)$, 
$\bar{P}_{i j}^{\varepsilon}=\bar{P}_{i j}^{0}+\varepsilon \bar{P}_{i j}^{1}+o(\varepsilon)$,

$\bar{u}_{i}^{\varepsilon}=\bar{u}_{i}^{0}+\varepsilon \bar{u}_{i}^{1}+o(\varepsilon)$,

for $i, j=1,2,3$. Substituting the change of variables (56-58) and the expansion (91) into the equilibrium equations of the interphase (third equation in (90)), one obtains

$\frac{1}{\eta^{ \pm}} \hat{\mathbf{P}}_{i 3,3}^{0}=0 \quad$ in $B$,

$\hat{\mathbf{P}}_{i 3,3}^{1}=-\eta^{ \pm} \hat{\mathbf{P}}_{i \alpha, \alpha}^{0}$ in $B$,

where the $+(-)$ sign applies in the part of $B$ with $z_{3}>0$ $\left(z_{3}<0\right)$. The first conditions implies that the stress components $\hat{\mathbf{P}}_{i 3}^{0}$ are constant with respect to $z_{3}$ in the rescaled interphase. By enforcing the continuity of the stress field at the interfaces between the adhesive and the adherents, Eq. (95) leads to the condition

$\left[\mathbf{P}^{0} \mathbf{e}_{3}\right]=\mathbf{0}$,

where, as the usual notation rule, $[f]$ denotes the jump across the surface $S$ of a function $f$ defined on the limit configuration obtained for $\varepsilon \rightarrow 0$.

Next, we substitute the modified change of variables (56-58) and the expansions $(91,93)$ into the constitutive equation of the interphase (last equation in (90)). This gives a set of conditions (at the orders $-3,-2$ and -1 ) all equivalent to the requirement that

$\hat{\mathbf{u}}_{, 3}^{0} \otimes \mathbf{e}_{3}=0$.

This condition in turn implies that the displacement is constant with respect to $z_{3}$ in the interphase and, enforcing the continuity of the displacement field at the interfaces between the adhesive and the adherents, it gives

$\left[\mathbf{u}^{0}\right]=\mathbf{0}$.

Substituting Eq. (98) into the condition arising from the (rescaled) constitutive equation of the interphase at order 0 , the following condition is obtained:

$\hat{\mathbf{P}}^{0}=\left(\mathbf{I}+\hat{\mathbf{H}}^{0}\right)\left\{\mu\left(\hat{\mathbf{H}}^{0}+\left(\hat{\mathbf{H}}^{0}\right)^{T}+\left(\hat{\mathbf{H}}^{0}\right)^{T} \hat{\mathbf{H}}^{0}\right)+\frac{1}{2} \lambda\left(\left(\mathbf{I} \cdot \hat{\mathbf{H}}^{0}\right)+\frac{1}{2}\left|\hat{\mathbf{H}}^{0}\right|^{2}\right) \mathbf{I}\right\}$,

with

$\hat{\mathbf{H}}^{0}=\hat{\mathbf{u}}_{, \alpha}^{0} \otimes \mathbf{e}_{\alpha}+\frac{1}{\eta^{ \pm}} \hat{\mathbf{u}}_{, 3}^{1} \otimes \mathbf{e}_{3}$.

Substituting (100) into (95) gives $\left(\hat{s}_{33}^{0} \mathbf{I}+\left(\mathbf{I}+\hat{\mathbf{H}}^{0}\right) \mathbf{K}^{33}\left(\mathbf{I}+\hat{\mathbf{H}}^{0}\right)^{T}\right) \hat{\mathbf{u}}_{, 33}^{1}=\mathbf{0}$,

with

$\hat{s}_{33}^{0}:=(2 \mu+\lambda)\left(\frac{1}{\eta^{ \pm}} \hat{u}_{3,3}^{1}+\frac{1}{\left(\eta^{ \pm}\right)^{2}} \frac{1}{2}\left|\hat{\mathbf{u}}_{, 3}^{1}\right|^{2}\right)+\lambda\left(\hat{u}_{\alpha, \alpha}^{0}+\frac{1}{2}\left|\hat{\mathbf{u}}_{, \alpha}^{0}\right|^{2}\right)$.

In view of Eq. (102) and under the assumption that

$\operatorname{det}\left(\hat{s}_{33}^{0} \mathbf{I}+\left(\mathbf{I}+\hat{\mathbf{H}}^{0}\right) \mathbf{K}^{33}\left(\mathbf{I}+\hat{\mathbf{H}}^{0}\right)^{T}\right) \neq 0$,

it is obtained that

$\hat{\mathbf{u}}_{, 33}^{1}=\mathbf{0}$.

This implies that $\hat{\mathbf{u}}_{3}^{1}$ is independent of $z_{3}$ and thus it admits the representation

$\hat{\mathbf{u}}^{1}(\overline{\mathbf{x}})=\left[\left[\hat{\mathbf{u}}^{1}\right]\right](\overline{\mathbf{x}}) z_{3}+<\hat{\mathbf{u}}^{1}>(\overline{\mathbf{x}})$.

In view of Eqs. $(105,106)$, one has that $\hat{\mathbf{H}}^{0}$ and $\hat{\mathbf{P}}^{0}$ are also independent of $z_{3}$. Thus, integrating (96) with respect to $z_{3}$ in the rescaled interphase gives

$\left[\left[\hat{\mathbf{P}}^{1} \mathbf{e}_{3}\right]\right]=-\eta \operatorname{div}_{p} \hat{\mathbf{P}}^{0}$,

with $\eta=\frac{1}{2}\left(\eta^{-}+\eta^{+}\right)$and the notation $\operatorname{div}_{p}$ indicates the divergence in the plane of the interphase, e.g.

$\operatorname{div}_{p} \mathbf{P}=\left(\mathbf{P e}_{1}\right)_{, 1}+\left(\mathbf{P e}_{2}\right)_{, 2}$.

To summarize, perfect interface conditions are obtained at the order zero and the nonlinear imperfect interface conditions (100) and (107) are obtained at the order 1. The imperfect interface conditions prescribe the jumps $\left[\left[\hat{\mathbf{P}}^{1}\right]\right] \mathbf{e}_{3}$, $\left[\left[\hat{\mathbf{u}}^{1}\right]\right]$, which can be related to the corresponding jumps $\left[\mathbf{P}^{1}\right] \mathbf{e}_{3},\left[\mathbf{u}^{1}\right]$ across the surface $S$ on the limit configuration obtained for $\varepsilon \rightarrow 0$ via matching conditions analogous to Eqs. (62)-(65) except for substituting the Cauchy stress $\sigma_{i j}$ with the Piola-Kirchhoff stress $P_{i j}$.

At the first two orders, the complete problems for the equivalent hard Saint Venant-Kirchhoff interface can be written as:

$\begin{cases}P_{i, j}^{0}+f_{i}=0 & \text { in } \Omega_{ \pm}, \\ P_{i j}^{0} n_{j}=g_{i} & \text { on } \Gamma_{g}, \\ u_{i}^{0}=0 & \text { on } \Gamma_{u}, \\ P_{i j}^{0}=\left(\delta_{i k}+u_{i, k}^{0}\right)\left(2 \mu^{ \pm}\left(u_{k, j}^{0}+u_{j, k}^{0}+\frac{1}{2} u_{h, k}^{0} u_{h, j}^{0}\right)+\lambda^{ \pm}\left(u_{l, l}^{0}+\frac{1}{2}\left|\mathbf{u}_{l}^{0}\right|^{2}\right) \delta_{k j}\right) & \text { in } \Omega_{ \pm}, \\ {\left[\mathbf{P}_{i j}^{0}=\mathbf{0}\right.} & \text { on } S, \\ {\left[u_{i}^{0}\right]=0} & \text { on } S .\end{cases}$ 


$$
\begin{cases}P_{i j, j}^{1}=0 & \text { in } \Omega_{ \pm}, \\ P_{i j}^{1} n_{j}=0 & \text { on } \Gamma_{g}, \\ u_{i}^{1}=0 & \text { on } \Gamma_{u}, \\ P_{i j}^{1}=\left(\delta_{i k}+u_{i, k}^{1}\right)\left(2 \mu^{ \pm}\left(u_{k, j}^{1}+u_{j, k}^{1}+\frac{1}{2} u_{h, k}^{1} u_{h, j}^{1}\right)+\lambda^{ \pm}\left(u_{l, l}^{1}+\frac{1}{2}\left|\mathbf{u}_{l}^{1}\right|^{2}\right) \delta_{k j}\right) & \text { in } \Omega_{ \pm}, \\ {\left[\mathbf{P}_{i 3}^{1}\right]=\eta\left(\operatorname{div}_{p} \mathbf{P}_{i 3}^{0}\right)_{i}+<<<\mathbf{P}_{i 3,3}^{0}>>>} & \text { on } S, \\ \mathbf{P}_{i 3}^{0}=\left(\left(\mathbf{I}+\mathbf{H}^{0}\right)\left\{\mu\left(\mathbf{H}^{0}+\left(\mathbf{H}^{0}\right)^{T}+\left(\mathbf{H}^{0}\right)^{T} \mathbf{H}^{0}\right)+\frac{1}{2} \lambda\left(\left(\mathbf{I} \cdot \mathbf{H}^{0}\right)+\frac{1}{2}\left|\mathbf{H}^{0}\right|^{2}\right) \mathbf{I}\right\}\right)_{i 3} & \text { on } S, \\ \mathbf{H}_{i j}^{0}=\left(\mathbf{u}_{, \alpha}^{0} \otimes \mathbf{e}_{\alpha}+\frac{1}{\eta^{ \pm}}\left(\left[\mathbf{u}_{i}^{1}\right]+<<<u_{i, 3}^{0}>>>\right) \otimes \mathbf{e}_{3}\right)_{i j} & \text { on } S .\end{cases}
$$

\section{A simple numerical example}

\subsection{Generalities}

In the following, numerical procedures are presented and a numerical example is treated. Two cases are considered: a soft cracked interface (Kachanov theory) and a hard cracked interface (dilute estimate), as presented in Sect. 4. In order to simplify the notations, only two dimensional problems are considered. For the soft interface, Eq. (41) is solved. For the case of a hard interface, Eqs. (50) and (51) are solved. The equilibrium problem (51) is written as

$$
\begin{cases}\operatorname{div} \boldsymbol{\sigma}^{1}=\mathbf{0} & \text { in } \Omega_{ \pm} \\ \boldsymbol{\sigma}^{1} \mathbf{n}=\mathbf{0} & \text { on } \Gamma_{g} \\ \mathbf{u}^{1}=\mathbf{0} & \text { on } \Gamma_{u} \\ \boldsymbol{\sigma}^{1}=\mathbf{a}_{ \pm} \mathbf{e}\left(\mathbf{u}^{1}\right) & \text { in } \Omega_{ \pm}^{0} \\ {\left[\mathbf{u}^{1}\right]=\mathbf{C}_{1}\left(\boldsymbol{\sigma}^{0} \mathbf{n}\right)+\mathbf{C}_{2}\left(\mathbf{u}^{0}\right)_{, 1}+<<\mathbf{u}_{, 3}^{0}>>} & \text { on } S \\ {\left[\boldsymbol{\sigma}^{1} \mathbf{n}\right]=\mathbf{C}_{3}\left(\boldsymbol{\sigma}^{0} \mathbf{n}\right)_{, 1}+\mathbf{C}_{4}\left(\mathbf{u}^{0}\right)_{, 11}} & \text { on } S\end{cases}
$$

where

$\mathbf{C}_{1}=\left(\begin{array}{cc}c_{1} & 0 \\ 0 & c_{2}\end{array}\right), \mathbf{C}_{2}=\left(\begin{array}{cc}0 & -1 \\ c_{3} & 0\end{array}\right), \mathbf{C}_{3}=\left(\begin{array}{cc}0 & c_{3} \\ -1 & 0\end{array}\right), \mathbf{C}_{4}=\left(\begin{array}{cc}c_{4} & 0 \\ 0 & 0\end{array}\right)$. with $\quad c_{1}=\frac{1}{\mu_{12}}, \quad c_{2}=\frac{E_{1}(1-v)-2 E_{2} v_{12}^{2}}{E_{1} \cdot E_{2}(1-v)}, \quad c_{3}=\frac{v_{12}}{1-v}$, $c_{4}=\frac{E_{1}}{1-v^{2}}$.

\subsection{Formulations of soft and hard interface problems}

Soft interface problem The soft interface problem at order zero (41) is variational and it can be written in the weak form:

$\int_{\Omega_{ \pm}} \mathbf{a}_{ \pm} \mathbf{e}(\mathbf{u}) \cdot \mathbf{e}(\mathbf{v}) d x+\int_{S}[\mathbf{v}] \cdot \mathbf{K}[\mathbf{u}] d s=\int_{\Omega_{ \pm}} \mathbf{f} \mathbf{v} d x$.

for all $\mathbf{v}^{ \pm} \in\left\{\mathbf{v} \in H^{1}(\Omega): \mathbf{v}=\mathbf{0}\right.$ on $\left.\Gamma_{u}\right\}$.

Next, using standard finite element on each subdomain, and a "flat" finite element on $S$ with all its nodes on $S$, half of them related to $\Omega_{-}$and the other half related to $\Omega_{+}$, it is possible to write a rigidity matrix of this problem that is invertible, and with standard error estimates (see for example $[8,31]$ for more details).

Hard interface problem Firstly, the interface problem at order 0 (see for example equation (50)) is standard since the continuity of the displacement and of the constraints across the interface are required. The problem (111) is no longer straightforward since discontinuities both in the displacement and in the stress vector fields have to be imposed. For the sake of simplicity, we denote by $\mathbf{D}^{0}=\mathbf{C}_{1}\left(\boldsymbol{\sigma}^{0} \mathbf{n}\right)+\mathbf{C}_{2}\left(\mathbf{u}^{0}\right)_{1}-\frac{1}{2}\left(\mathbf{u}^{0}\left(x_{1}, 0^{+}\right)+\mathbf{u}^{0}\left(x_{1}, 0^{-}\right)\right)$and $\mathbf{S}^{0}=\mathbf{C}_{3}\left(\boldsymbol{\sigma}^{0} \mathbf{n}\right)_{, 1}+\mathbf{C}_{4}\left(\mathbf{u}^{0}\right)_{11}$ the imposed jumps, computed for the solution at order 0 .

Then, the problem (111) has to be split into two parts, one for the jump in the displacements, the other for the jump in the constraints.

More precisely, we write $\mathbf{u}^{ \pm}=\mathbf{w}^{ \pm}+\mathbf{z}^{ \pm}$where the unknowns $\mathbf{z}^{ \pm}$and $\mathbf{w}^{ \pm}$solve the problems

$\begin{cases}\operatorname{div} \boldsymbol{\sigma}\left(\mathbf{z}^{ \pm}\right)=\mathbf{0} & \text { in } \Omega_{ \pm} \\ \boldsymbol{\sigma}\left(\mathbf{z}^{ \pm}\right) \mathbf{n}=\mathbf{0} & \text { on } \Gamma_{g} \\ \mathbf{z}^{ \pm}=\mathbf{0} & \text { on } \Gamma_{u} \\ \boldsymbol{\sigma}\left(z^{ \pm}\right)=\mathbf{a}^{ \pm} \mathbf{e}\left(\mathbf{z}^{ \pm}\right) & \text {in } \Omega_{ \pm} \\ \mathbf{z}^{+}=\mathbf{D}^{0} & \text { on } S \\ \mathbf{z}^{-}=\mathbf{0} & \text { on } S\end{cases}$

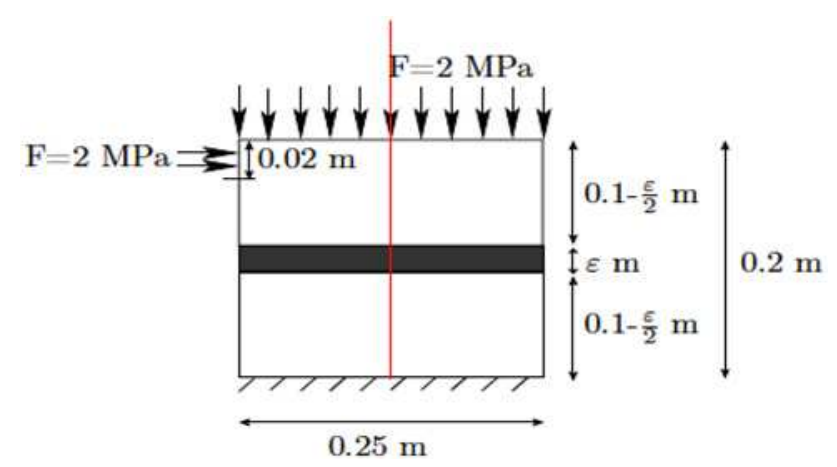

Fig. 3 Geometry of the problem $(\varepsilon=0$ for the interface problem) 
Table 1 Elastic properties of bricks and mortar

\begin{tabular}{lll}
\hline & Young modulus $(\mathrm{MPa})$ & Poisson ratio \\
\hline Bricks & 14 & 0.2 \\
Undamaged mortar & 11 & 0.2 \\
\hline
\end{tabular}

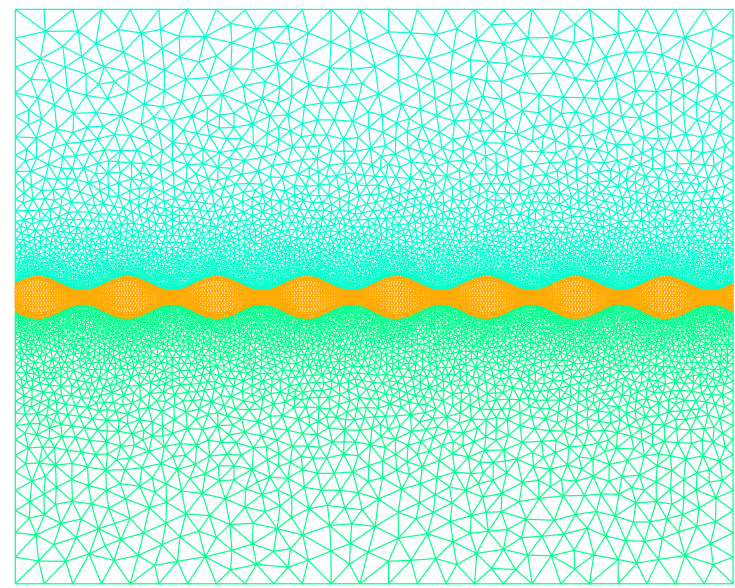

$Z^{Y} \times$

Fig. 4 Geometry and mesh of the problem of Fig. 3 with a smoothlyrough interphase $(\varepsilon=0.01 \mathrm{~m})$

$$
\begin{cases}\operatorname{div} \boldsymbol{\sigma}\left(\mathbf{w}^{ \pm}\right)=\mathbf{0} & \text { in } \Omega_{ \pm} \\ \boldsymbol{\sigma}\left(\mathbf{w}^{ \pm}\right) \mathbf{n}=\mathbf{0} & \text { on } \Gamma_{g} \\ \mathbf{w}^{ \pm}=\mathbf{0} & \text { on } \Gamma_{u} \\ \boldsymbol{\sigma}\left(\mathbf{w}^{ \pm}\right)=\mathbf{a}^{ \pm} \mathbf{e}\left(\mathbf{w}^{ \pm}\right) & \text {in } \Omega_{ \pm} \\ {[\mathbf{w}]=\mathbf{0}} & \text { on } S \\ {[\boldsymbol{\sigma}(\mathbf{w}) \mathbf{n}]=\mathbf{S}^{0}-[\boldsymbol{\sigma}(\mathbf{z}) \mathbf{n}]} & \text { on } S\end{cases}
$$

since $\quad[\mathbf{w}]=\mathbf{w}^{+}-\mathbf{w}^{-}=[\mathbf{u}]-\mathbf{z}^{+}+\mathbf{z}^{-}=\mathbf{D}^{0}-\mathbf{D}^{0}=\mathbf{0}$. The two first problems defined in Eq. (112) both in $\Omega_{+}$and $\Omega_{-}$are standard and they can be solved simultaneously by using a standard finite element method (let us notice that $\mathbf{z}^{-}$is vanishing on $\Omega_{-}$and thus there is no problem to solve on this domain, and $\mathbf{z}^{+}$can be viewed as a prolongation of $\mathbf{D}^{0}$ on $\Omega_{+}$). Equation (113) is solved by using the Nitsche's method:

$$
\begin{aligned}
\int_{\Omega_{+} \cup \Omega_{-}} \boldsymbol{\sigma}\left(\mathbf{w}^{ \pm}\right) \cdot \mathbf{e}\left(\mathbf{v}^{ \pm}\right) d x & +\int_{S}(<<\boldsymbol{\sigma}(\mathbf{w}) \mathbf{n}>>\cdot[\mathbf{v}]+[\mathbf{w}] \cdot<<\boldsymbol{\sigma}(\mathbf{v}) \mathbf{n}>>) d S \\
& +\frac{\beta}{h} \int_{S}[\mathbf{w}] \cdot[\mathbf{v}] d S=-\int_{S} \mathbf{S}^{0} \cdot<<\mathbf{v}>>d S,
\end{aligned}
$$

for all $\mathbf{v} \in\left\{H^{1}(\Omega): \gamma(\mathbf{v})=0\right.$ on $\left.\partial \Omega \backslash S\right\}$, where $h$ is the size of the smallest element of the finite element discretization of $\Omega_{ \pm}$, and $\beta>0$ is a fixed number that must be sufficiently large to ensure the stability of the method.

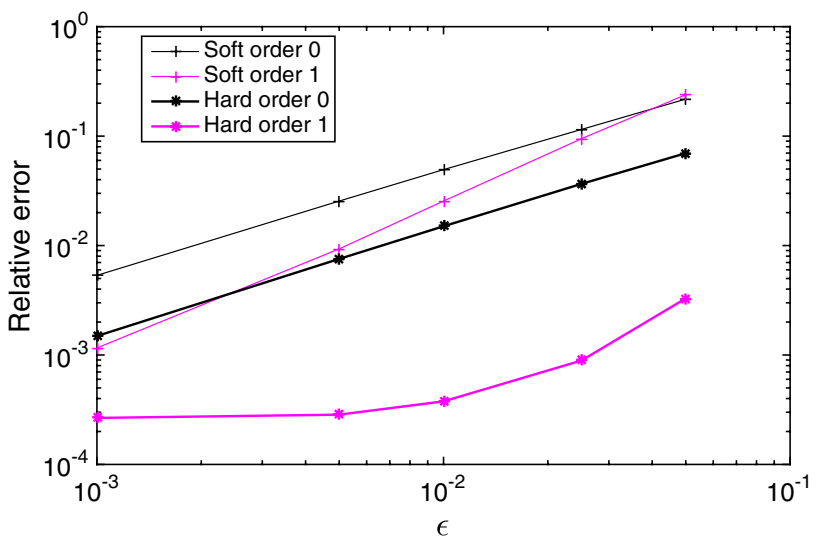

Fig. 5 Convergence of the various modeling to a reference solution when $\varepsilon$ tends to 0

Table 2 Representative coefficients for the Kachanov and the Dilute Model for various values of the crack length $(L=0.2 \mathrm{~m}, \varepsilon=0.01 \mathrm{~m})$

\begin{tabular}{lllll}
\hline $\begin{array}{l}\text { Crack length } \\
(\mathrm{m})\end{array}$ & $l=0.002$ & $l=0.005$ & $l=0.01$ & $l=0.02$ \\
\hline $\begin{array}{l}\text { Kachanov } \\
\text { model: }\end{array}$ & & & & \\
$K_{N}\left(\frac{N}{m^{3}}\right)$ & $1.3428 \cdot 10^{5}$ & $2.1484 \cdot 10^{4}$ & $5.3711 \cdot 10^{3}$ & $1.3428 \cdot 10^{3}$ \\
$K_{T}\left(\frac{N}{m^{3}}\right)$ & $1.2085 \cdot 10^{5}$ & $1.9336 \cdot 10^{4}$ & $4.8340 \cdot 10^{3}$ & $1.2085 \cdot 10^{3}$ \\
$\begin{array}{l}\text { Dilute model: } \\
C_{\rho}\end{array}$ & 0.9918 & 0.9485 & 0.7904 & 0.0988 \\
\hline
\end{tabular}

This formulation, known as the Nitsche's method [32], is equivalent to (113). In particular, the solutions of (114) are weak solutions of (113) (see [1, 8, 47] for the complete study of this method and for a priori and a posteriori error estimates).

\subsection{Numerical results}

In this section, we present some numerical results on an academic example. The geometry of the problem is given in Fig. 3. The composite structure consists of bricks glued with damaged mortar. The problem is treated in plane stresses. In all the computations bellow, P2 triangular finite elements (conforming piecewise quadratic Lagrangian finite elements) are used. These elements are characterized by six nodes (one on each vertex and one on the middle of each edge).

All materials are considered as isotropic and their mechanical parameters are listed in Table 1. 


\subsection{Roughness}

In this first study, we consider a roughness equal to $\eta\left(x_{2}\right)=\sin \left(16 \pi x_{2}\right)$ for $x_{2} \in[0,0.25] \quad$ (see Fig. 4 for $\varepsilon=0.01 \mathrm{~m})$.

For each values of $\varepsilon$ ranging from $\varepsilon=0.05 \mathrm{~m}$ to $0.001 \mathrm{~m}$, we calculate the $L^{2}$ relative error between the solution computed with the three phases (as in Fig. 4) and a solution computed with the modelings proposed in the previous sections (soft/hard interface, at order 0 or 1). The results are plotted on Fig. 5.

In Fig. 5, one can observe that, since the relative rigidity of the mortar and the brick is small, the hard interface modeling provides better results, both at order 0 and 1 . For that modeling, the relative error is of order $0(\varepsilon)$ when considering the order 0 and it converges rapidly to $10^{-4}$ when considering the order 1 . For the soft interface law, one can observe the convergence rate is close to $0(\varepsilon)$ at order 0 and to $0\left(\varepsilon^{1.5}\right)$ at order 1 .

\subsection{Microcracks}

For the applications with microcracks, we have considered a mortar thickness equal to $\varepsilon=0.01$ and values of crack length ranging from $\frac{l}{L}=0.1$ (very damaged) to $\frac{l}{L}=0.01$.
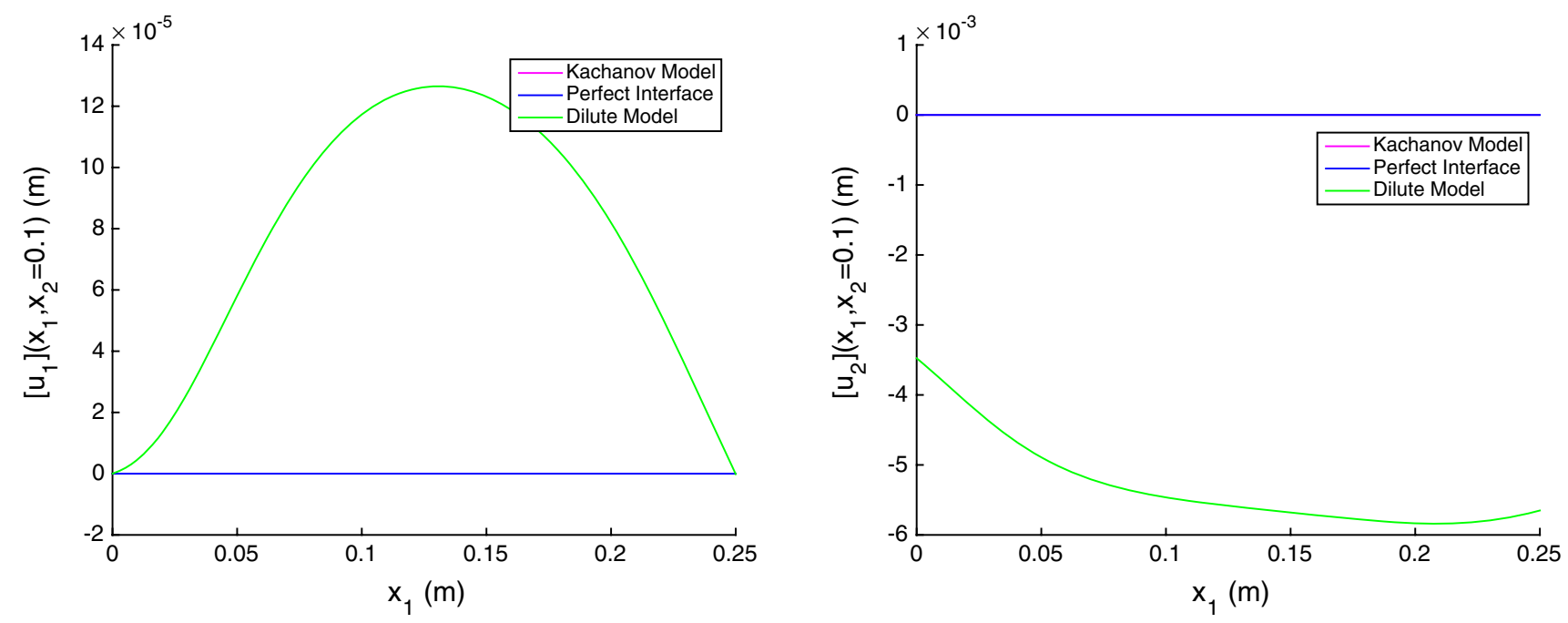

Fig. 6 Jumps in the displacement along the interface $\left[u_{1}\right]\left(x_{1}, x_{2}=0.1\right)$ and $\left[u_{2}\right]\left(x_{1}, x_{2}=0.1\right)$ (right), for $\varepsilon=0.01 \mathrm{~m}$ and $l=0.002 \mathrm{~m}$ (the $t w o$ first curves overlap)
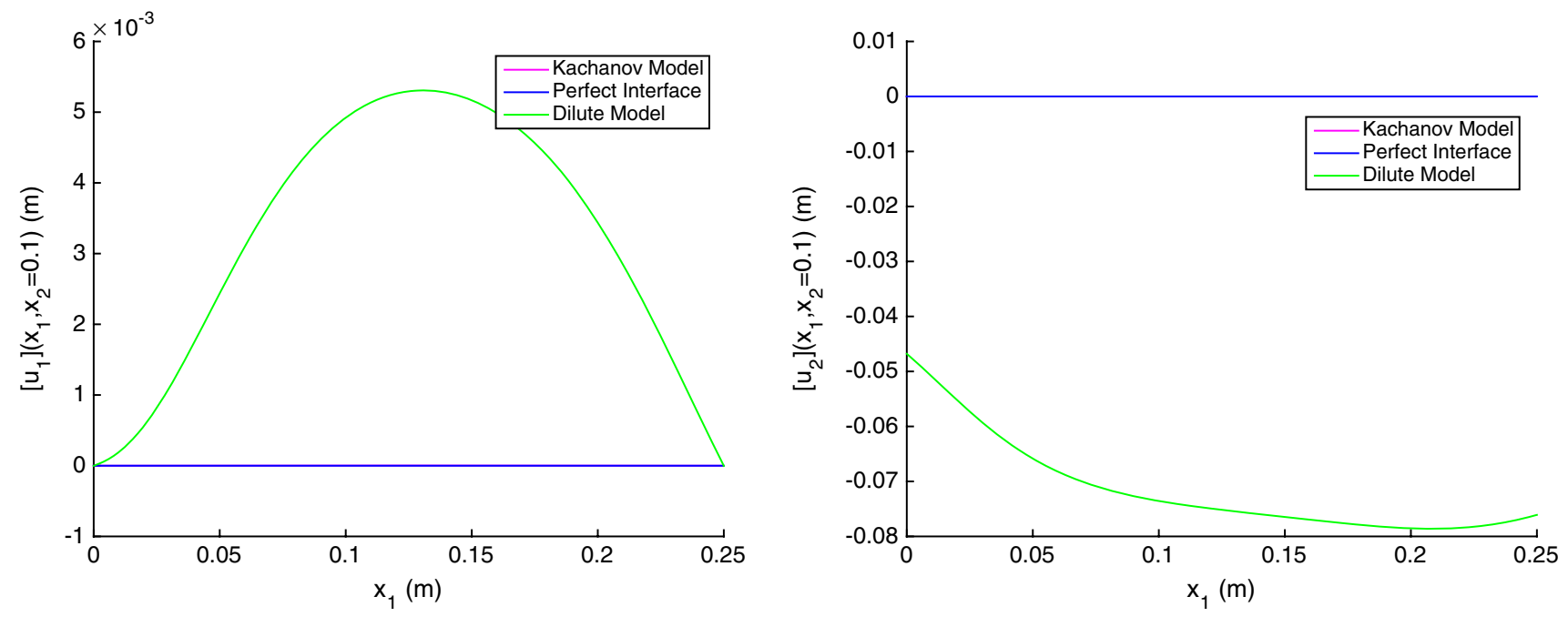

Fig. 7 Jumps in the displacement along the interface $\left[u_{1}\right]\left(x_{1}, x_{2}=0.1\right)$ and $\left[u_{2}\right]\left(x_{1}, x_{2}=0.1\right)$ (right), for $\varepsilon=0.01 \mathrm{~m}$ and $l=0.02 \mathrm{~m}$ (the $t w o$ first curves overlap) 

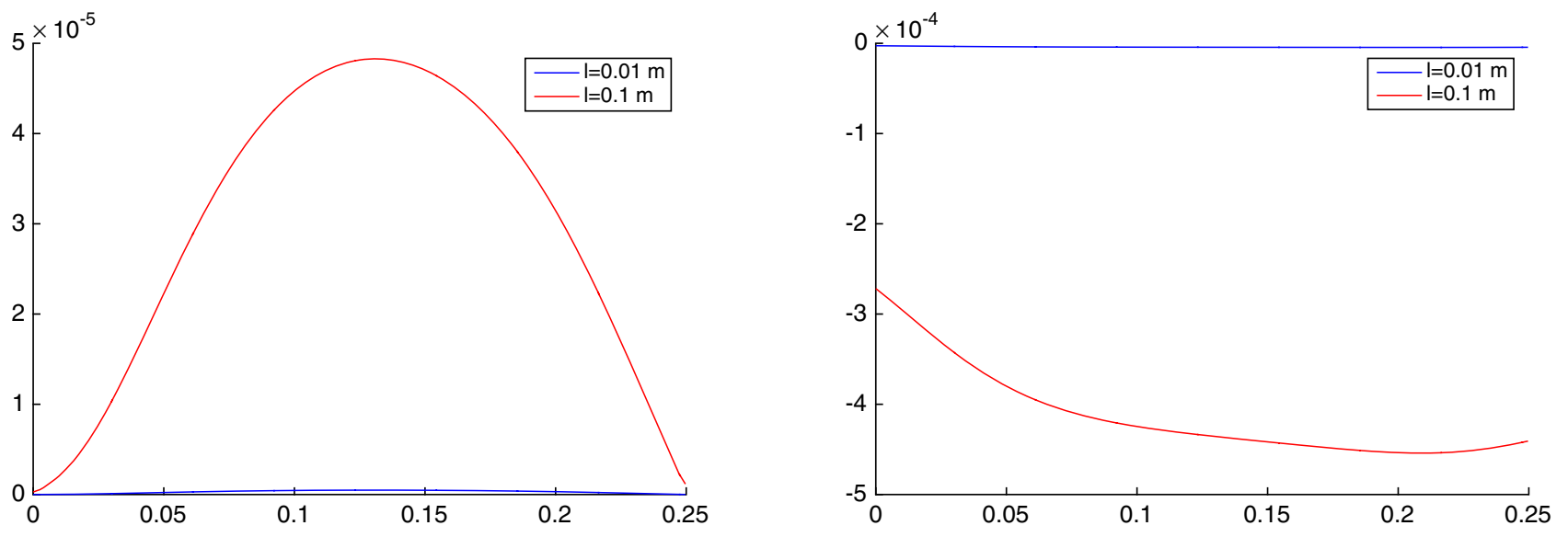

Fig. 8 Jumps in the displacement along the interface $\left[u_{1}\right]\left(x_{1}, x_{2}=0.1\right)$ and $\left[u_{2}\right]\left(x_{1}, x_{2}=0.1\right)($ right $)$, for $\varepsilon=0.01, l=0.01 \mathrm{~m}$ and $l=0.1 \mathrm{~m}$

For each values of the crack length, Table 2 shows the representative coefficients for each theory. Let us notice that, for an undamaged mortar, we have $K_{N}=K_{T}=+\infty$ for the Kachanov model and $C_{\rho}=1$ for the diluted model.

Data in Table 2 indicate that, when the crack length decreases, the rigidity of the interface increases for the Kachanov model and the diluted model becomes closer to the undamaged model.

We also note that for $l$ larger than $0.02 \mathrm{~m}$, the coefficient $C_{\rho}$ becomes negative, an occurrence mechanically not admissible. On the contrary, for the Kachanov model, such physical restriction does not exist.

Finally, some solutions for extremal values of the parameters $\varepsilon$ and $\frac{l}{L}$ are presented. In the first set of plots, we have used the Kachanov theory, imposing the continuity of the constraints and of the displacements across the interface, and the diluted model to obtain the displacements in both vertical and horizontal directions.

If we study more precisely the jumps in the displacements along the interface, we can observe that these jumps are smaller for $l=0.002 m$ (see Fig. 6) than for $l=0.01 \mathrm{~m}$ (see Fig. 7). Moreover, the jumps calculated for the Kachanov Model are very small in the considered range of values for $l$.

We then present some results obtained with the Kachanov model with larger values of the crack length, values that are unreachable with the dilute model. In Fig. 8, we can observe that the jumps in the displacements along the interface increase strongly when the length of the crack increases.

\section{Conclusion and perspectives}

In this paper, a general methodology aimed at modeling thin interphases and based on asymptotic expansions is presented. A review of classical "soft" and "hard" imperfect interfaces models in elasticity is first proposed. The methodology is generalized in a simple way to introduce smooth roughness and micro-cracks. In the first case, it is shown that a change in a step of the asymptotic procedure, i.e. the rescaling, allows to introduce the roughness in the interface model. In the second case, micro-cracks are introduced by a simple modification of the elastic stiffness tensor. Next, a nonlinear constitutive equation for the thin interphase is introduced.

In the last part of the paper, a simple and efficient numerical method is described and an academic example is treated. In particular, it is shown how roughness and the presence of macro-cracks inside the interphase material can influence the interface model.

The results of present paper are expected to find applications in different contexts, especially for modeling the complex behavior of nonlinear or piezoelectric adhesives $[14,25,46]$, of imperfect bonding in composites [5, 26, $28,33]$, and quasi-brittle interfaces in masonry structures $[36,38,39]$.

Furthermore, the proposed model has been generalized to introduce damage evolution, seen as the micro-crack length evolution $[3,38,39]$, and it has been implemented in a commercial FEM-based software within some applications in masonry structures [38, 39]. As a perspective, the proposed methodology could be applied to the study of more general nonlinear constitutive equations, e.g., in biomechanical applications.

Acknowledgements RR thanks the financial support from ENDIF through the "Fondo per necessità di base della ricerca - 2014".

\section{References}

1. Becker R, Hansbo P, Stenberg R (2010) A finite element method for domain decomposition with non-matching grids. ESAIM M2AN 37(2):209-225 
2. Benveniste $Y$, Miloh T (2001) Imperfect soft and stiff interfaces in two-dimensional elasticity. Mech Mater 33(6):309-323

3. Bonetti E, Bonfanti G, Lebon F, Rizzoni R (2017) A model of imperfect interface with damage. Meccanica (in press)

4. Bornert PG, Bretheau T, Gilormini P (2001) Homogénéisation en mécanique des matériaux, Tome 1: Matériaux aléatoires élastiques et milieux périodiques. Hermes Sciences, Paris

5. Challamel N, Girhammar UA (2011) Boundary-layer effect in composite beams with interlayer slip. J Aerosp Eng 24(2):199-209

6. Ciarlet PG (1988) Mathematical elasticity: three-dimensional elasticity, vol 1. North-Holland

7. Dacorogna B (2008) Direct methods in the calculus of variations. Appl Math Sci 78. Springer, New York

8. Dumont S, Goubet O, Ha-Duong T, Villon P (2006) Meshfree methods and boundary conditions. Int J Numer Meth Eng 67:989-1011

9. Dumont S, Lebon F, Rizzoni R (2014) An asymptotic approach to the adhesion of thin stiff films. Mech Res Com 58:24-35

10. Dacorogna B, Marcellini P (1995) Existence of minimizers for non-quasiconvex integrals. Arch Ration Mech Anal 131:359-399

11. Fouchal F, Lebon F, Raffa ML, Vairo G (2014) An interface model including cracks and roughness applied to masonry. Open Civ Eng J 8:263-271

12. Geymonat G, Krasucki F, Lenci S (1999) Mathematical analysis of a bonded joint with a soft thin adhesive. Math Mech Sol 4(2):201-225

13. Hashin $Z$ (2002) Thin interphase/imperfect interface in elasticity with application to coated fiber composites. J Mech Phys Solids 50(12):2509-2537

14. Ivanov IV, Velchev DS, Kneć M, Sadowski T (2011) Computational models of laminated glass plate under transverse static loading. In: Altenbach H, Eremeyev VA (eds)"Shell-line structures", non-classical theories and applications. Adv Struct Mat, Springer, Berlin, pp 469-490 (2011)

15. Klarbring A (1991) Derivation of the adhesively bonded joints by the asymptotic expansion method. Int J Eng Sci 29:493-512

16. Lebon F, Ould Khaoua A, Licht C (1998) Numerical study of soft adhesively bonded joints in finite elasticity. Comp Mech 21:134-140

17. Lebon F, Rizzoni R (2008) Asymptotic study on a soft thin layer: the non-convex case. Mech Adv Mat Struct 15:12-20

18. Lebon F, Rizzoni R (2010) Asymptotic analysis of a thin interface: the case involving similar rigidity. Int J Eng Sci 48:473-486

19. Lebon F, Rizzoni R (2011) Asymptotic behavior of a hard thin linear elastic interphase: an energy approach. Int J Sol Struct 48:441-449

20. Lebon F, Rizzoni R (2013) Modeling a hard, thin curvilinear interface. Discr Cont Dyn Syst Ser S 6(6):1569-1586

21. Lebon F, Rizzoni R, Ronel-Idrissi S (2004) Asymptotic analysis of some non-linear soft thin layers. Comp Struct 82:1929-1938

22. Lebon F, Ronel-Idrissi S (2004) Asymptotic analysis of Drucker-Prager and Mohr-Coulomb soft thin interfaces. Steel Comp Struct 4:133-147

23. Lebon F, Zaittouni S (2010) Asymptotic modelling of interfaces taking contact conditions into account: asymptotic expansions and numerical implementation. Int J Eng Sci 48:111-127

24. Le Dret H, Raoult A (1995) The quasiconvex envelope of the Saint Venant-Kirchhoff stored energy function. Proc R Soc Edinb 125A:1179-1192

25. Lenci S, Clementi F, Warminski J (2015) Nonlinear free dynamics of a two-layer composite beam with different boundary conditions. Meccanica 50(3):675-688

26. Li YD, Xiong T, Dong L (2015) A new interfacial imperfection coupling model (IICM) and its effect on the facture behavior of a layered multiferroic composite: anti-plane case. Eur J Mech A Solids 52:26-36

27. $\mathrm{Li} \mathrm{Z}$ (1996) Existence of minimizers and microstructure in nonlinear elasticity. Nonlinear Anal Theor 27(3):297-308

28. Lopez-Realpozo JC, Rodriguez-Ramos R, Guinovart-Diaz R, Bravo-Castillero J, Otero JA, Sabina FJ, Lebon F, Dumont S, Sevostianov I (2014) Effective elastic shear stiffness of a periodic fibrous composite with non-uniform imperfect contact between the matrix and the fibers. Int J Solids Struct 51(6):1253-1262

29. Mardare C (2010) Existence of minimizers for the pure displacement problem in nonlinear elasticity, "Alexandru Myller" mathematical seminar. Romania 1329:181-190

30. Mauge C, Kachanov M (1994) Effective elastic properties of an anisotropic material with arbitrarily oriented interacting cracks. J Mech Phys Solids 42:561-584

31. Nairn JA (2007) Numerical implementation of imperfect interfaces. Comp Mat Sci 40:525-536

32. Nitsche J (1974) Convergence of nonconforming methods. In: Mathematical aspects of finite elements in partial differential equations. Proc. Sympos. Math. Res. Center, University of Wisconsin, Madison. Academic Press, New York, pp 15-53

33. Orifice A, Mancusi G, Dumont S, Lebon F (2016) An experimental/numerical study on the interfacial damage of bonded joints for fibre-reinforced polymer profiles at service conditions. Technologies. doi:10.3390/technologies4030020

34. Pelissou C, Lebon F (2009) Asymptotic modeling of quasi-brittle interfaces. Comp Struct 87:1216-1223

35. Ponte Castaneda M, Willis J (1995) The effect of spatial distribution on the effective behavior of composite materials and cracked media. J Mech Phys Sol 43:1919-1951

36. Raffa ML, Lebon F, Rizzoni R (2016) On modelling brick/mortar interface via a St. Venant-Kirchhoff orthotropic soft interface. Part I: theory. Int J Mason Res Innov 1(2):142-164

37. Raffa ML, Lebon F, Vairo G (2016) Normal and tangential stiffnesses of rough surfaces in contact via an imperfect interface model. Int J Sol Struct 87:245-253

38. Raffa ML, Lebon F, Rizzoni R (2017) On modelling brick/mortar interface via a St. Venant-Kirchhoff orthotropic soft interface. Part II: in silico analysis. Int J Mason Res Innov (in press)

39. Raffa ML, Lebon F, Rizzoni R (2017) Derivation of a model of imperfect interface with finite strains and damage by asymptotic techniques. Application to masonry structures. Meccanica (in review)

40. Rekik A, Lebon F (2010) Identification of the representative crack length evolution in a multi-level interface model for quasibrittle masonry. Int J Solids Struct 47(22-23):3011-3021

41. Rekik A, Lebon F (2012) Homogenization methods for interface modeling in damaged masonry. Adv Eng Softw 46(1):35-42

42. Rizzoni R, Lebon F (2012) Asymptotic analysis of an adhesive joint with mismatch strain. Eur J Mech A Sol 36:1-8

43. Rizzoni R, Lebon F (2013) Imperfect interfaces as asymptotic models of thin curved elastic adhesive interphases. Mech Res Comm 51:39-50

44. Rizzoni R, Dumont S, Lebon F (2015) On Saint Venant-Kirchhoff imperfect interfaces. Int J Non-Linear Mech 89:101-115

45. Rizzoni R, Dumont S, Lebon F, Sacco S (2014) Higher order model for soft and hard elastic interfaces. Int J Solids Struct 51:4137-4148

46. Serpilli M (2014) Asymptotic analysis of a multimaterial with a thin piezoelectric interphase. Meccanica 49(7):1641-1652

47. Stenberg R (1995) On some techniques for approximating boundary conditions in the finite element method. J Comput Appl Math 63:139-148

48. Tsukrov I, Kachanov M (2000) Effective moduli of an anisotropic material with elliptical holes of arbitrary orientational distribution. Int J Solids Struct 37(41):5919-5941 